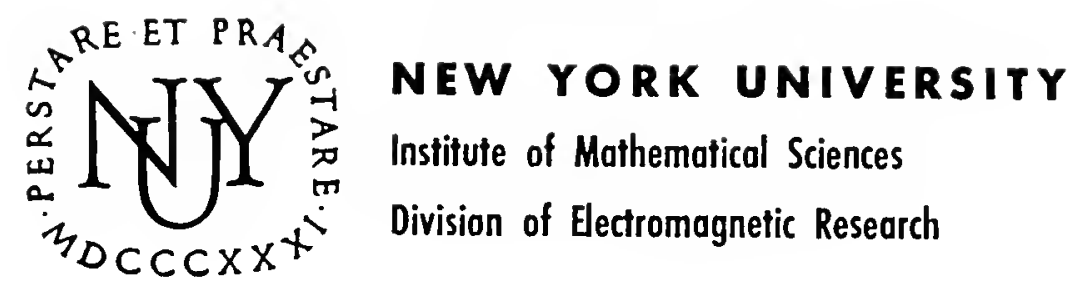

RESEARCH REPORT No. HSN-2

\title{
Analytical Theory of Turbulent Diffusion
}

\author{
P. H. ROBERTS
}

$N$

$2 \gamma$

ก j

Contract No. N(onr)-285(33)

J U N E , 1960 


\section{NEW YORK UNIVERSITY}

Institute of Mathematical Sciences

Division of Electromagnetic Research

Research Report No. HSN-2

Analytical Theory of Turbulent Diffusion

P. H. ROBERTS ${ }^{\dagger}$

P. H. Robets

P.H. Roberts

This work was supported by the Office of Naval Research under Contract No. Nonr-285(33)

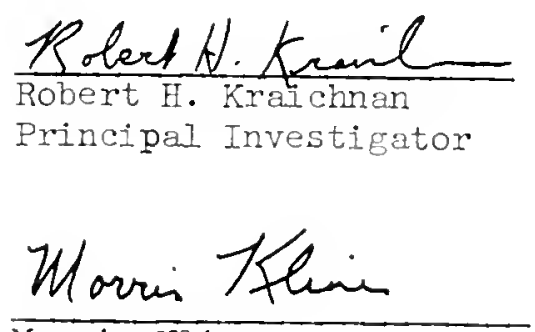

$t_{\text {permanent address: }}$

Dept. of Physics, King's College, Newcastle-upon-Tyne, England 



\section{Abstract}

Recently Kraichnan (1959) has propounded a theory of homogeneous turbulence, based on a novel perturbation method, that leads to closed equations for the velocity covariance. In the present paper, this method is applied to the theory of turbulent diffusion and closed equations are derived for the probability distributions of the positions of marked fluid elements released in a turbulent flow.

Two topies are discussed in detail. The first is the probabllity distribution, at time $t$, of the displacement of an element from 1 ts initial position. In homogeneous flows, this distribution is found to resemble that for classical dffusion but with a variable coefficient of diffusion which is proportional to $v_{0}^{2} t$ for $t \ll \ell / v_{0}$ and which approaches a constant value $\doteq \ell v_{0}$ for $t \gg l / v_{0} \quad\left(\ell=\right.$ macroscale, $v_{0}=r . m . s$. turbulent velocity).

The second topic treated is the joint probability distribution of the displacements of two fluid elements. Particular attention is focused upon the probability distribution of relative displacement, i.e. Richardson's distance-neighbour function. This is found to be Gaussian for separations $r$ which are large $(\gg \ell)$. For smaller separations $(r<<\ell)$, its behavior at high Reynolds numbers is found to be quite well expressed in terms of a variable diffusion coefficient $K(r, t)$, as suggested by Richardson (1926). For all but extremely short times, $K(r, t)$ is found to depend only on $r$ and on the form of the inertial range spectrum $E(k)$. on assuming $\mathrm{E}(\mathrm{k}) \propto \mathrm{v}_{0}^{2} \boldsymbol{\ell}(\mathrm{kl})^{-3 / 2}$ as results from Kraichnan's approximation (1959), one finds $K(r) \propto v_{0} \ell(r / \ell)^{3 / 2}$. On the basis of similarity 
arguments of the Kolmogorov type, which give $E(k) \propto v_{0}^{2} l(k l)^{-5 / 3}$, one finds $K(r) \propto v_{0} \ell(r / \ell)^{4 / 3}$ as, in fact, Richardson originally proposed. The dispersion $\left\langle r^{2}\right\rangle$ is proportional to $\ell^{2}\left(v_{0} t / l\right)^{4}$ on Kraichnan's theory; while $\left\langle r^{2}\right\rangle \propto \ell^{2}\left(v_{0} t / l\right)^{3}$ on the similarity theory. This illustrates that the behavior of $\left\langle r^{2}\right\rangle$ is very sensitive to the spectrum.

\section{Table of Contents}

Page

1. Introduction

2. Diffusion from a fixed source

2.1 Methods of approach

2.2 Exact results for short times

2.3 An integro-differential equation for $\mathrm{G}\left(\mathrm{x},\left.\mathrm{t}\right|_{\mathrm{x}_{0}}, \mathrm{t}_{0}\right)$

2.4 Solution of the integro-differential equation for short times

2.5 Solution for large times

3. Relative diffusion

3.1 Formulation of problem: exact results for short times

3.2 Integral equations for $G\left(\underset{m}{x}, t ; y_{w}, s \mid x_{m}, t_{0} ; y_{w}, s_{0}\right)$

3.3 Solution of equations for short times

3.4 Solutions for large and Intermediate times

Appendix A: Derivation of equation (2.26)

44

Appendix B: Derivation of equation (3.18)

56

References . 2 5

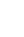
9 


\section{INTRODUCTION}

The aim of the theory of turbulent diffusion is to determine in a statistical sense the migration of marked particles as they are carried along with a turbulent flow. Like molecular motion in a dilute gas of discrete particles, turbulent diffusion is a linear process 11 the convected particles have no reaction on the flow; $1 . e$. the probab1lity distribution for the position of a marked particle in space obeys the superposition rule and changes in time according to a linear equation. Unlike classical molecular motion, the motion of nelghborlng fluld elements in a continuum is correlated, although one expects that over distances large compared to the macroscale $\ell$ of the turbulence this correlation 1 s weak and that elements separated by such distances move almost Independently. Furthermore, unlike classical molecular diffusion, turbulent diffusion is not a Markoff process. However, one expects that

over times large compared to $\ell / v_{0}$, where $v_{0}$ is the root mean square fluta veloclty, the fluid elements wlll suffer many essentially uncorrelated deflections by the energy contalning eddies and that accordingly their motion over such long times wlll be almost Brownian. Under such c1rcumstances, one expects that the spread of marked particles carried by the fluid w1ll indeed resemble classical diffusion, and that it will be possible to define a coefflclent of eddy diffusivity. An analytical basis for these qualitative observations is given in Sec. 2.

Particles which start out simultaneously at nearby points have closely simflar historles in any one realization of the turbulent flow (and over times which are not too long). For such times, their relative motion 18 unaffected by eddies whose spatial scale is large compared to 
the initial separation; such eddies give nearly equal displacements to the two points. The change in the separation $r$ is governed by the smaller eddies, particularly those whose length scale is of the same order as $r$. Thus, in a flow of high Reynolds number, we expect that while the particles are separated by a distance appropriately small compared to $\ell$ their relative diffusion will be governed by the inertial range spectrum of the turbulent flow, and will be unaffected by the structure of the energy containing eddies. An analytical basis for these surmises is given in sec. 3, and a form is proposed for the variable diffusion coefficient introduced by Richardson (1926).

\section{DIFFUSION FROM A FIXED SOURCE}

\subsection{Methods of approach}

There are two main ways of attempting to give an analytical framework to the qualitative arguments of Sec. 1. In the Lagrangian framework (as distinct from the Eulerian approach to be described presently), probability distributions are defined for the displacements, velocities, etc., of given marked particles, and the relationships between them are studied. For example, let $G\left(x_{m}, t \mid x_{0}, t_{0}\right) d x$ be the probability that a fluid particle lying at the point $x_{0}$ at time $t_{0}$ should, at the later time $t$, lie within a volume $d x$ at the point $x_{-}$. Let $V\left(x_{m}, u_{m},\left.t\right|_{x_{0}}, t_{0}\right) d_{x} d u_{m}$ be the probability that this same particle should at that time lie within dx and have a velocity between $u$ and $u+d u$. Then it is not difficult to show that

$$
\frac{\partial}{\partial t} G\left(\underset{\sim}{x},\left.t\right|_{\sim 0}, t_{0}\right)+\frac{\partial}{\partial x_{i}} \int V\left(\underset{\sim}{x}, \underline{u},\left.t\right|_{\sim 0} ^{x_{0}}, t_{0}\right) u_{i} d u=0 .
$$

(In 2.1 and elsewhere, we use the summation convention.) 
Through the hydrodynamical equations, it is possible to derive a similar, though far more involved equation, relating $V$ to another probability function, and this, in its tum, to yet another. It would be necessary to close this hierarchy of equations in some way in order to obtain from it an evaluation of $G\left(\underset{x}{x}, t \mid x_{m}, t_{0}\right)$.

The second approach (advocated by G.K. Batchelor, 1952b; see also W.H. Re1d, 1955; P.H. Roberts, 1957) involves reformulating the problem of finding Lagrangian probability functions as one of determining certain Eulerian moments. A passive scalar quantity $\psi(\underset{m}{x}, t)$ is introduced which satisfles the equation

$$
\frac{\partial}{\partial t} \psi(\underline{x}, t)+\frac{\partial}{\partial x_{i}}\left[\psi(\underline{x}, t) u_{i}(x, t)\right]=0,
$$

where $u_{1}(x, t)$ is the velocity field. This quantity is therefore carried by the turbulent fluid, but does not affect its motion. It is clear that if we take

$$
\psi\left(\underset{\sim}{x}, t_{0}\right)=\delta\left(x_{-m}-x_{0}\right)
$$

where $\delta(x)$ is the three dimensional Dirac $\delta$-function, then

$$
\psi(\underline{x}, t)=\delta(\underline{x}-\underline{x})
$$

where $x_{-t}$ is the position at time $t$ (in this particular realization of u) of the fluid element which was initially at $\underset{m 0}{x}$. By averaging over all realizations, we see that the solution of

$$
\frac{\partial}{\partial t}\langle\psi(x, t)\rangle+\frac{\partial}{\partial x_{i}}\left\langle\psi(x, t) u_{i}(x, t)\right\rangle=0,
$$




\section{4.}

which satisfies the initial condition

$$
\left\langle\psi\left(x, t_{0}\right)\right\rangle=\delta\left(x_{m}^{x}-x_{0}\right)
$$

is

$$
\langle\psi(\underset{w}{x}, t)\rangle=G\left(x_{m}^{x}, t \mid x_{0}, t_{0}\right)
$$

Through a simflar though more complicated equation, $\left\langle\psi u_{i}\right\rangle$ is related to higher moments, and so on. Again, we have an hilerarchy of equations which must be closed in some way in order to evaluate $G\left(x, t \mid x_{0}, t_{0}\right)$.

In the present paper, we adopt the second of these two approaches and employ an approximation method for closing the equations devised by Kraichnan (1959). However, before presenting this approxtmate analysis, we shall derive some results which are asymptotically exact for small $t_{0} \cdot$

\subsection{Exact results for short times}

For $t-t_{0} \ll \ell / v_{0}$, the fluid particles are simply swept from their points of origin with whatever velocity the turbulent fluid happens to have at the moment of their release; $1 . e$.

$$
\left.V\left(\underline{x}_{-},\left.t\right|_{-0}, t_{0}\right)=P\left[\underline{u}_{-0}, t_{0}\right)\right] \delta\left[\left(\underline{x}_{-x_{0}}\right)-\underline{u}\left(t-t_{0}\right)\right]
$$

where $P\left[\underline{u}\left(x_{0}, t_{0}\right)\right]$ is the probability distribution function (p.d.f.) of $u$ at position $x_{0}$ and time $t_{0}$. Thus, by $(2.1)$, or by inspection

$$
G\left(x,\left.t\right|_{x_{0}}, t_{0}\right)=\frac{1}{\left(t-t_{0}\right)^{3}} P\left(\frac{x-x_{0}}{t-t_{0}}\right)
$$


This result can also be deduced from the formal solution

$$
\begin{aligned}
\psi(x, t) & =\psi\left(x_{m}, t_{0}\right)-\int_{t_{0}}^{t} d t^{\prime} \frac{\partial}{\partial x_{i}}\left[u_{i}\left(x_{w}, t^{\prime}\right) \psi\left(x, t_{0}\right)\right] \\
& +\int_{t_{0}}^{t} a t^{\prime} \frac{\partial}{\partial x_{i}}\left\{u_{i}\left(x, t^{\prime}\right) \int_{t_{0}}^{t^{\prime}} d t^{\prime \prime} \frac{\partial}{\partial x_{j}}\left[u_{j}\left(x, t^{\prime \prime}\right) \psi\left(x, t_{0}\right)\right]\right\}-\ldots,
\end{aligned}
$$

which one obtains from (2.2) by integration and 1teration. When $t-t_{0} \ll \ell / v_{0}$, then $\underline{u}(x, t) \doteq u\left(x_{m}, t_{0}\right)$, and it follows that

$$
\begin{gathered}
\psi(x, t) \doteq\left[1-\left(t-t_{0}\right) u_{1}\left(x_{0}, t_{0}\right) \frac{\partial}{\partial x_{1}}+\frac{1}{2 !}\left(t-t_{0}\right)^{2} u_{1}\left(x_{0}, t_{0}\right) u_{j}\left(x_{0}, t_{0}\right) \frac{\partial^{2}}{\partial x_{i} \partial x_{j}}\right. \\
-\ldots] \psi\left(x_{-}, t_{0}\right) \cdot
\end{gathered}
$$

Hence, by $(2.6)$ and $(2.7)$,

$$
\begin{aligned}
& G\left(x_{m},\left.t\right|_{w_{0}}, t_{0}\right) \doteq\left[I-\left(t-t_{0}\right)\left\langle u_{1}\left(x_{0}, t_{0}\right)\right\rangle \frac{\partial}{\partial x_{1}}\right. \\
& \left.+\frac{1}{2 !}\left(t-t_{0}\right)^{2}\left\langle u_{i}\left(x_{0}, t_{0}\right) u_{j}\left(x_{0}, t_{0}\right)\right\rangle \frac{\partial^{2}}{\partial x_{1} \partial x_{j}}-\ldots\right] \delta\left(x_{-\infty}^{x-x_{0}}\right) .
\end{aligned}
$$

That this is equivalent to (2.9) can most easily be seen by expressing the result in wave-vector space, writing

$$
G\left(x_{-},\left.t\right|_{x_{0}}, t_{0}\right)=\int \widetilde{G}\left(k,\left.t\right|_{-0}, t_{0}\right) e^{i k \cdot\left(x-x_{0}\right)} d a_{0}
$$


Then (2.12) is equivalent to

$$
\begin{aligned}
\tilde{G}\left(k_{w}^{k}, t \mid x_{0}, t_{0}\right) & =\frac{1}{(2 \pi)^{3}}\left[1-1 k_{i}\left(t-t_{0}\right)\left\langle u_{i}\left(x_{0}, t_{0}\right)\right\rangle\right. \\
& \left.-\frac{1}{2 !} k_{1} k_{j}\left(t-t_{0}\right)^{2}\left\langle u_{i}\left(x_{0}, t_{0}\right) u_{j}\left(x_{0}, t_{0}\right)\right\rangle+\ldots\right] ; \\
& \tilde{G}\left(k, t \mid x_{0}, t_{0}\right)=\frac{1}{(2 \pi)^{3}} \tilde{P}\left[\left[-t_{0}\right)\right],
\end{aligned}
$$

where

$$
\widetilde{P}(\eta)=\int P\left[\underline{u}\left(x_{-0}, t_{0}\right)\right] e^{-i \underline{\eta} \cdot \underline{u}} d \underline{u}
$$

is the characteristic function for the distribution of velocity at $\mathrm{x}_{0}$ and $t_{0}$. Equation (2.9) is simply the inverse of (2.15).

This second method of establishing the behavior of $G$ at small times brings out some noteworthy features. If (2.12) is cut off after any finite number of terms, it implies that $G\left(x, t \mid \underline{x}_{0}, t_{0}\right)$ vanishes identically for non-zero $x_{-x} x_{0}$ on the other hand, if $(2.14)$ is cut off after a finite number of terms, the resulting expression for $\tilde{G}$ diverges for large $\mathrm{k}$, or large $t-t_{0}{ }^{\circ}$ One concludes that any reasonable approximate solution for the full space-function $G\left(x_{w},\left.t\right|_{x_{0}}, t_{0}\right)$ must include terms of orders of the formal expansion (2.10). Even for very short times the formal expansion is only useful because we happen to be able to sum it to all orders. 
However, if the moments

$$
\begin{aligned}
& \left\langle\Delta x_{1}\right\rangle=\int \Delta x_{1} G\left(x,\left.t\right|_{-0}, t_{0}\right) d x_{m}=i(2 \pi)^{3}\left[\frac{\partial}{\partial k_{i}} \tilde{G}\left(\underline{k},\left.t\right|_{x_{0}}, t_{0}\right)\right]_{k=0} \\
& \left\langle\Delta x_{1} \Delta x_{j}\right\rangle=\int \Delta x_{1} \Delta x_{j} G\left(\underline{x}_{-},\left.t\right|_{-0}, t_{0}\right) d x_{m}=-(2 \pi)^{3}\left[\frac{\partial^{2}}{\partial k_{i} \partial k_{j}} \widetilde{G}\left(\underline{k},\left.t\right|_{\sim 0}, t_{0}\right)\right]_{k=0}
\end{aligned}
$$

$\left(\Delta \underline{x}=x_{m}-x_{0}\right)$ are expanded by means of the formal solution, the resulting serles appear to converge for all $t-t_{0}$, although the convergence 1 s poor unless $t-t_{0} \ll \ell / v_{0}$. Equation $(2.14)$ shows that for small $t-t_{0}$,

$$
\left\langle\Delta x_{1} \Delta x_{j}\right\rangle=U_{i j}\left(x_{m o}, t_{0} ; x_{0}, t_{0}\right)\left(t-t_{0}\right)^{2},
$$

where

$$
U_{1 j}\left(x, t ; x_{0}, t_{0}\right)=\left\langle u_{1}(x, t) u_{j}\left(x_{m}, t_{0}\right)\right\rangle
$$

is the velocity covariance. In the isotroplc case, therefore,

$$
\left\langle\Delta x_{i} \Delta x_{j}\right\rangle=v_{1}^{2} \delta_{1 j}\left(t-t_{0}\right)^{2}
$$

where $v_{1}^{2}$ is the mean square of any component of veloctty at position $x_{0}$ and time $t_{0}$.

There is fairly strong experimental evidence (see, for example, Batchelor, 1953, Ch. 8) that $\mathrm{P}$ almost always is closely Gaussian. In this case, $G\left(x,\left.t\right|_{m o}, t_{0}\right)$ must be closely Gaussian also for short times. 
Then by (2.14) and (2.9) we have

$$
\tilde{G}\left(k_{m}, t \mid{\underset{\rightarrow 0}{x}}_{0}, t_{0}\right)=\frac{1}{(2 \pi)^{3}} \exp \left[-\frac{1}{2} U_{i j}\left(x_{w_{0}}, t_{0} ; x_{0}, t_{0}\right) k_{i} k_{j}\left(t-t_{0}\right)^{2}\right],
$$

and

$$
\begin{aligned}
G\left(x,\left.t\right|_{x_{0}}, t_{0}\right)= & \frac{1}{(2 \pi)^{3 / 2}\left(t-t_{0}\right)^{3}\left(\operatorname{det} U_{1 j}\right)^{1 / 2}} \\
& x \exp \left[-\frac{1}{2} u_{i j} \Delta x_{i} \Delta x_{j}\left(t-t_{0}\right)^{-2}\right],
\end{aligned}
$$

where $u_{i j}$ is the cofactor of $U_{i j}\left(x_{m}, t_{0} x_{w_{0}}, t_{0}\right)$ and det $U_{i j}$ denotes the determinant of these quantities. In the isotropic case,

$$
\tilde{G}\left(k,\left.t\right|_{m 0}, t_{0}\right)=\frac{1}{(2 \pi)^{3}} \exp \left[-\frac{1}{2} k^{2} v_{1}^{2}\left(t-t_{0}\right)^{2}\right]
$$

and

$$
G\left(x,\left.t\right|_{m o}, t_{0}\right)=\frac{1}{\left[2 \pi v_{1}^{2}\left(t-t_{0}\right)^{2}\right]^{3 / 2}} \exp \left[-\left(x-x_{m_{0}}\right)^{2} / 2 v_{1}^{2}\left(t-t_{0}\right)^{2}\right] .
$$

\subsection{An integro-differential equation for $G\left(x, t \mid x_{0}, t_{0}\right)$}

When $t-t_{0}$ is not small compared to $\ell / v_{0}$, the approximation (2.11) to the formal solution (2.10) is Invalid since it is no longer legitimate to ignore the space and time variation of $u_{i}(x, t)$. It is nevertheless possible to effect a partial summation of (2.1) which includes terms from every order in the expansion, and is such that the resulting expression for $\widetilde{G}$ converges 
for large $\mathrm{k}$. The integrul equation for this approximate form of $G$ is derived in fppendix A in two ways. The first mutes use of Kraichnan's direct interaction approximation (Kraichnan, 1359). In the second method, the same result is derived by liscaring or retaining tems in the formal expansion according to a certain selection criterion. The terms retalned are of all order. Each of these methods supposes tnat the velocity field is spatially homogeneous, but this restriction can be removed by the application of a more seneral method tue to Kraichnan (1960). The final result, for the case where the mean field $\langle u(x, t)\rangle$ vanishes, is the equation

$$
\begin{aligned}
\frac{\partial}{\partial t} G\left(x, t \mid x_{0}, t_{0}\right) & =\int_{t_{0}}^{t} d t^{\prime} \int d x^{\prime} U_{i j}\left(x_{,}, t ; x_{0}, t_{0}\right) \\
& \times \frac{\partial}{\partial x_{i}} G\left(x_{\infty}^{x}, t \mid x_{m}^{\prime}, t^{\prime}\right) \frac{\partial}{\partial x_{j}^{\prime}} G\left(x_{m}^{\prime}, t \mid x_{m}, t_{0}\right) .
\end{aligned}
$$

Here, as later, the fluid velocity is supposed incompressible:

$$
\partial u_{i}(x, t) / \partial x_{i}=0
$$

(The compressible case can be treated by similar methods.) In the case of statistically homogeneous and stationary flows, we may write

$$
\left.\begin{array}{l}
G\left(x, t \mid x_{m}, t_{0}\right)=G\left(x_{m}-x_{0}, t-t_{0}\right), \\
U_{i j}\left(x, t ; x_{m}, t_{0}\right)=U_{i j}\left(x-x_{0}, t-t_{0}\right),
\end{array}\right\}
$$


and, upon a partial integration, (2.23) becomes

$\frac{\partial}{\partial t} G(x, t)=\frac{\partial^{2}}{\partial x_{i} \partial x_{j}} \int_{0}^{t} d t^{\prime} \int d x^{\prime} U_{i j}\left(x_{w j}^{\prime}, t^{\prime}\right) G\left(x^{\prime}, t^{\prime}\right) G\left(x-x^{\prime}, t-t^{\prime}\right)$.

Multiplying (2.26) by $x_{i} x_{j}$ and Integrating the right-hand side by parts, we find

$\frac{\partial}{\partial t}\left\langle x_{i} x_{j}\right\rangle=\int x_{i} x_{j} \frac{\partial}{\partial t} G(x, t) d x=2 \int_{0}^{t} d t^{\prime} \int d x^{\prime} U_{i j}\left(x^{\prime}, t^{\prime}\right) G\left(x^{\prime}, t^{\prime}\right) ;$ i.e.

$$
\left\langle x_{i} x_{j}\right\rangle=2 t \kappa_{i j}(t)
$$

where the variable "eddy diffusivity" tensor $k_{i j}(t)$ is defined by

$$
\kappa_{i j}(t)=\frac{1}{t} \int_{0}^{t} d t^{\prime}\left(t-t^{\prime}\right) \int d x_{m}^{\prime} U_{i j}\left(x_{n=m}^{\prime}, t^{\prime}\right) G\left(x^{\prime}, t^{\prime}\right)
$$

For short times $\left(t<\ell / v_{0}\right),(2.27)$ and $(2.28)$ agree with $(2.17)$, if we assume $G\left(x^{\prime}, t^{\prime}\right)$ is negligible unless $\left|x^{\prime}\right| \ll \ell$. Then,

$$
\kappa_{i j} \doteq \frac{1}{2} t U_{i j}(0,0), \quad t \rightarrow u \text {. }
$$

For laxge times $\left(t \gg \ell / v_{0}\right)$, 
$k_{i j} \doteq \int_{0}^{\infty} a t^{\prime} \int a x^{\prime} U_{i j}\left(x_{m}^{\prime}, t^{\prime}\right) G\left(x_{m}^{\prime}, t^{\prime}\right)=\frac{1}{(2 \pi)^{3}} \int_{0}^{\infty} d t^{\prime} \int d k \tilde{U}_{i j}\left(k, t^{\prime}\right) \widetilde{G}\left(-k, t^{\prime}\right)$

Let us assume that the diffusion for time $z l / v_{0}$ is dominated by the energy-containing eddies. Then it is reasonable to suppose that the integral in (2.30) should depend only on the parameters $\ell$ and $v_{0}$, whence, by dimensional reasoning, we must have

$$
\kappa_{i j} \rightarrow \text { constant of order } \ell v_{0}, \quad t \rightarrow \infty
$$

In the isotropic case, we have (

$k_{i j}=k \delta_{i j}, \quad k=\frac{4 \pi}{3} \int_{0}^{t} d t^{\prime}\left(t-t^{\prime}\right) \int_{0}^{\infty} d x^{\prime} x^{\prime} U_{i 1}\left(x^{\prime}, t^{\prime}\right) G\left(x^{\prime}, t^{\prime}\right)$.

Equations (2.27) and (2.28), and their generalizations for flow which are not steady or homogeneous, imply that the Lagrangian correlation function $\left\langle u_{i}(t) u_{j}\left(t_{0}\right)\right\rangle_{L}($ ef. Taylor, 1921) is

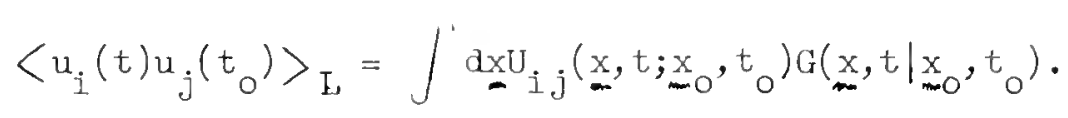

This should be compared to the exact result

$$
\left\langle u_{i}(t) u_{j}\left(t_{0}\right)\right\rangle_{L}=\int d_{m}\left\langle u_{i}\left(x_{m}^{x}, t\right) u_{j}\left(x_{m}, t_{0}\right) \psi\left(x_{m}, t \mid x_{m}, t_{0}\right)\right\rangle,
$$


where $\psi\left(x, t \mid x_{m}, t_{0}\right)$ denotes the unaveraged Green's function, $1 . e$. the solution of (2.2) satisfying (2.3). Equation (2.32) clearly may be obtained from (2.33) on the assumption that the $\psi$ and $u$ fields are uncorrelated (see also Corrsin, 1960) ${ }^{*}$. However, if one decides to use an assumption of this kind, the results depend very much on what stage of the analysis is chosen for its application. For example, had we assumed that $\psi$ and $\underline{u}$ are uncorrelated in (2.2), we would have obtained the absurd result $\partial\langle\psi(x, t)\rangle / \partial t=0$. Moreover, if the approximation

$\left\langle u_{i}(x, t) u_{j}\left(x_{m 0}, t_{0}\right) \psi\left(x, t \mid x_{m}, t_{0}\right)\right\rangle=U_{i j}\left(x, t ; x_{\infty}, t_{0}\right) G\left(x,\left.t\right|_{\infty}, t_{0}\right)$

is used to close the hierarchy of equations which arise from (2.2) and (2.3), the result is not consistent with (2.32) (cf. Roberts, 1957). Thus, the direct-1nteraction approximation is consistent with (2.34) only if $(2.34)$ is used in a particular way.

\subsection{Solution of the integro-differential equation for short times}

For $t \ll \ell / v_{0}$, we may assume that the $G$ factors in the integrand of (2.23) are negligible unless $x-x_{0}$ and $x_{m}^{\prime-x}$ are both small comparea with $\ell$. Thus, $U_{i j}\left(x_{m}^{\prime}, t^{\prime}, x_{0}, t_{0}\right)$ in the integrand may be replaced by $U_{i j}\left(x_{m}, t_{0}, x_{m o}, t_{0}\right)$ or, in the isotropic case, by $v_{I}{ }^{2} \delta_{i j}$. It follows that

$\frac{\partial}{\partial t} G(x, t)=v_{1}^{2} \nabla^{2} \int_{0}^{t} d t^{\prime} \int d x_{m}^{\prime} G\left(x^{\prime}, t^{\prime}\right) G\left(x-x^{\prime}, t-t^{\prime}\right)$.

Saffman (unpublished) has used the approximate result (2.32) to derive an estimate for $k$ (see Sec. 2.5 below). 
Taking the Fourier transform

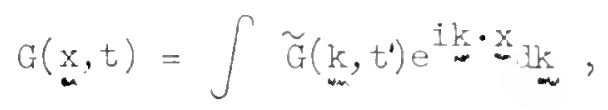

we find

$$
\frac{\partial}{\partial t} \tilde{G}(k, t)=-(2 \pi)^{3} v_{1} 2 k^{2} \int_{0}^{t} a t^{\prime} \tilde{G}\left(\underset{m}{k}, t^{\prime}\right) \tilde{G}\left(\underset{m}{k}, t-t^{\prime}\right) .
$$

By taking the Laplace transform of (2.36), or by using a result of watson (1944, Sec. 12.2, eq. 5), we find

$$
\tilde{G}(k, t)=\frac{1}{(2 \pi)^{3}} \frac{J_{1}\left(2 k v_{1} t\right)}{k v_{1} t}, \quad(k=|k|) .
$$

Inverting by using a further result of Watson (1944, Sec. 13.42, eq. (4)), we find

$$
G(x, t)= \begin{cases}\frac{1}{\left(2 \pi v_{1} t\right)^{2}}\left[{ }^{4} v_{1}{ }^{2} t^{2}-x^{2}\right]^{-1 / 2}, & \text { if } x<2 v_{1} t, \\ 0 & , \text { if } x>2 v_{1} t .\end{cases}
$$

The corresponding probability aistribution $D\left(x_{1}, t\right)$ for aisplacement along the $x_{I}$-axis (whose airection may be chosen arbitrarily) is related to $G(x, t)$ by

$$
\partial D(x, t) / \partial x=-2 \pi x G(x, t)
$$


and so, in the present case,

$$
D\left(x_{1}, t\right)= \begin{cases}\frac{1}{2 \pi\left(v_{1} t\right)^{2}}\left[4 v_{1}{ }^{2}{ }^{2}-x_{I}^{2}\right]^{1 / 2}, & \text { if } x_{1}<2 v_{I} t, \\ 0 & , \text { if } x_{I}>2 v_{1} t .\end{cases}
$$

Both $G(x, t)$ and $D\left(x_{1}, t\right)$ are everywhere non-negative, but they are zero beyond a distance of $2 v_{1} t$ from the source. That this behavior is not restricted to small times can be seen in a rough way from the fact that $k^{3 / 2} \widetilde{G}(k, t)$ oscillates with wavelength $2 v_{1} t$ as $k \rightarrow \infty$. A more convincing demonstration can be given directly by induction from (2.26). One supposes that $G\left(x^{\prime}, t^{\prime}\right)$ vanishes when $\left|x^{\prime}\right|>2 v_{1} t^{\prime}$ for all times $t^{\prime} \leqq t^{\prime \prime}$ and, by approximating to (2.26) for points near the wave-front $|x|=2 v_{1} t^{\prime \prime}$, one can show that this implies that $G(x, t)$ vanishes for $|x|>2 v_{1} t$, where $t$ is infinitesimally greater than $t "$. The finite maximum "propagation speed" exhibited by (2.38) therefore persists for all times. This is also consistent with (2.9) which implies, in the present case, that the p.d.f. of velocity has a sharp cut-off at $u=2 v_{I}$. These properties are physically unreasonable. They arise in some way from the direct interaction approximation.

A related unrealistic feature of $(2.36)$ is that $G(x, t) \rightarrow \infty$ as $x \rightarrow 2 v_{l} t-0$. However, the singularity is weak, since

$$
\int_{x}^{2 v_{1} t+0} 4 \pi x^{2} G(x, t) d x \doteq \frac{4}{\pi}\left(2-\frac{x}{v_{I} t}\right)^{1 / 2}, x \rightarrow 2 v_{1} t-0 ;
$$


i.e. the singular outgoing wave front does not even carry a finite integrated probability. It is perhaps worth mentioning that this singular type of behavior is not unknown in random walk problems although it is generally smoothed away after a few steps. (For random walk in a plane, the distribution after two steps shows singularities, see Watson Sec. 13.48; Sec. 13.46, eq. (3); for random walk on a sphere similar results hold, see Roberts and Ursell, 1959, p. 320, and footnote.)

For a normally distributed velocity fleld, a comparison of (2.37) in the form

$$
\widetilde{G}(k, t)=\frac{1}{(2 \pi)^{3}} \sum_{0}^{\infty} \frac{(-1)^{n}\left(k v_{1} t\right)^{2 n}}{n !(n+1) !},
$$

with the exact solution (2.21) in the form

$$
\tilde{G}(k, t)=\frac{1}{(2 \pi)^{3}} \sum_{0}^{\infty} \frac{(-1)^{n}\left(k v_{1} t\right)^{2 n}}{2^{n} n !},
$$

shows that, for short times, the direct interaction approximation gives the second moments of $\mathrm{G}(\mathrm{x}, \mathrm{t})$ correctly. The fractional errors in the fourth, sixth, and $2 n^{\text {th }}$ moments are, respectively, $1 / 3,2 / 3$, and $\left[1-2^{n} /(n+1) !\right]$. This exhibits in another way the consequences of the effective cut-off in "propagation speed".

\subsection{Solution for large times}

Fot $t \gg \ell / v_{0}$, the diffusing particles will have suffered many displacements (statistically almost unrelated) from the energy containing eddies, and we may expect $G(\underset{w}{x}, t)$ to become close to a Gaussian distribution. This may be 
established directly (cf. Taylor, 1921) by expressing the moments of the distribution as integrals over Lagrangian velocity correlations. It can then be shown that, for times large compared to the Lagrangian correlation times (assuming these to be finite), these moments approach Gaussian values corresponding to an eddy diffusivity

$$
k_{i j}=\int_{0}^{\infty} d t^{\prime}\left\langle u_{i}\left(t^{\prime}\right) u_{j}\left(t_{0}\right)\right\rangle_{L} .
$$

In a like manner, for $t \gg \ell / v_{0}$, the distribution satisfying (2.26) becomes Gaussian with an eddy diffusivity

$$
k_{i j}=\int_{0}^{\infty} d t^{\prime} \int d x^{2} G\left(x^{i}, t^{i}\right) U_{i j}\left(x^{1}, t^{\prime}\right) .
$$

One way of proving this is to extend to the higher moments the arguments that led to (2.27). For example, it is easy to show from (2.26) that

$$
\begin{aligned}
\left\langle x_{i} x_{j} x_{k} x_{l}\right\rangle & =\left\langle x_{i} x_{j}\right\rangle\left\langle x_{k} x_{l}\right\rangle+\left\langle x_{i} x_{k}\right\rangle\left\langle x_{j} x_{l}\right\rangle+\left\langle x_{i} x_{l}\right\rangle\left\langle x_{j} x_{k}\right\rangle \\
& +2\left[\int_{0}^{t} d t^{\prime}\left(t-t^{\prime}\right) \int d x^{\prime} x_{i}^{\prime} x_{j}^{\prime} U_{k l}\left(x^{\prime}, t^{\prime}\right) G\left(x^{\prime}, t^{\prime}\right)+5 \text { similar terms }\right] .
\end{aligned}
$$

Assuming that expressions such as

$$
\frac{1}{t} \int_{0}^{t} d t^{\prime}\left(t-t^{\prime}\right) \int d x^{\prime} x_{i}^{\prime} x_{j}^{\prime} U_{k \ell}\left(x^{\prime}, t^{t}\right) G\left(x_{m}^{\prime}, t^{\prime}\right)
$$


converge as $t \rightarrow \infty$, we see that for $t \gg \ell / v_{0}$ the first three terms on the right-hand side of $(2.45)$ are of order $\left(\ell_{0} t\right)^{2}$ while the remainder are of order $\ell^{3} \mathrm{v}_{0} \mathrm{t}$ and therefore are comparatively neglibible. Thus the fourth orker cumulants can be neglected if $t \gg \ell / v_{0}$. Similar arguments hold in all orders and show that $G$ approaches nomality. This result may also be established by the following altemative method.

If $t \gg \ell / v_{0}$, the mean distance $\sqrt{\left\langle x^{2}\right\rangle}$ the particles will have travelled from their source and will be large compared to $\ell$ so that the length and time scales of $G(x, t)$ will be large compared to $\ell$ and $\ell / v_{0}$, respectively. Under these circumstances, the only regions of integration in (2.26) for which the integrand is appreciable are those for which $G\left(x-x^{\prime}, t-t^{\prime}\right)$ is approximately equal to $G(x, t)$. Thus, with $k_{i j}$ given by $(2.44)$, we have

$$
\frac{\partial G(x, t)}{\partial t}=k_{i j} \frac{\partial^{2} G(x, t)}{\partial x_{i} \partial x_{j}}
$$

since for such large times it is immaterial whether the upper limit of integration in (2.26) is $t$ or $\infty$. In the isotropic case (2.46) becomes

$$
\frac{\partial G(x, t)}{\partial t}=k \nabla^{2} G(x, t),
$$

where $k$ is given by (2.31) with $t$ replaced by $\infty$ 。

We may regard $(2.46)$ as the first term in a series of approximations based on expanding the term $G\left(x-x^{\prime}, t-t^{\prime}\right)$ in the integrand of (2.26) in a Taylor series about the point $x, t$, and we may apply an a posteriori check on the reasonableness of the approximation by verifying that, on substituting in the second term of this series the value of $G(x, t)$ derived 
from (2.46), this second term is small compared to either side of (2.46). We will refrain from giving the analysis, which is straightforward. It confirms that, if the relevant integrals converge, the necessary conditions for the validity of $(2.46)$ are

$$
t \gg \ell / v_{0}, \quad x \ll v_{0} t
$$

The second condition arises from the finiteness of the maximum propagation velocity which, as we have already seen, gives an artificial cut-off at the distance $2 v_{1} t$. We may expect that for the actual case in which this cut-off is not present the second of the conditions (2.48) would be unnecessary and that the distribution of particles would be Gaussian at all distances. In any event, when the first condition is satisfied, it is clear that the fraction of particles affected by the second condition is negligibly small.

It seems extremely likely, from the exact short-time result of Sec. 2.2 and our present results for large times, that $G(x, t)$ is nearly Gaussian for all times, and that the variable diffusion coefficients defined in Sec. 2.3 (cf. eqs. 2.27, 2.28, 2.31) will give useful estimates of the variance of the distribution at all times. On assuming a Gaussian form for $G(x, t)$, we obtain from (2.28) an integral equation for $k_{1 j}(t)$. The approximation of (2.33) by (2.32) has been proposed independently by P.G. Saffman (unpublished) in the homogeneous case. By assuming a Gaussian form for $G(x, t)$, and the isotropic form 


$$
\tilde{u}_{i j}(k, t)=\left[\delta_{1 j}-\frac{k_{i} k j}{k^{2}}\right] \frac{v_{i}^{2} k^{2}}{\pi^{3 / 2} 5} \exp \left\{\left[\frac{k^{2}}{k_{0}^{2}}+\frac{1}{2} v_{1} k^{2} t^{2}\right]\right\},
$$

he has obtained from the integral equation for $k_{i j}$ the estimate

$$
\kappa \sim 0.7 v_{1} / k_{0}, \quad(t \rightarrow \infty),
$$

where $\pi^{I / 2_{k}}{ }^{-1}$ is the Iongitudinal integral scale (cf. Batchelor, 1953, p.47).

\section{RELATIVE DIFFUSION}

\subsection{Formulation of problem: exact results for short times}

In this section, we study the correlation between the motion of two marked particles which are initially separated by a distance small compared to $\ell$. The choice of method is essentially that of Sec. 2.I and again we will adopt a formulation of terms of Eulerian moments. We introduce the passive scalar field $\psi_{1}(\underline{x}, t)$ for the first particle and $\psi_{2}(y, s)$ for the second particle, and we require that both fields satisfy (2.2). For $\psi_{I}(x, t)$, we take as initial condition

$$
\psi_{1}\left(x, t_{0}\right)=\delta\left(x-x_{0}\right),
$$

and, for $\psi_{2}(x, t)$, we take

$$
\psi_{2}\left(y_{m}, s_{0}\right)=\delta\left(y-y_{m}\right)
$$


Let us define a two particle Green's function by

$$
G\left(\underset{m}{x}, t ; y, s \mid x_{m}, t_{0} ; y_{0}, s_{0}\right)=\left\langle\psi_{1}(x, t) \psi_{2}(y, s)\right\rangle
$$

Clearly, since the two particles have identical properties,

$$
G\left(\underset{m}{x}, t ; y,\left.s\right|_{m} ^{x}, t_{0} ; y_{m}, s_{0}\right)=G\left(\underset{m}{y}, s ; x,\left.t\right|_{m o}, s_{0} ; x_{m}, t_{0}\right)
$$

In homogeneous steady turbulence, it depends only on difference times and difference coordinates. We shall then write it as

$$
G\left(\underset{w}{x-x_{0}}, t-t_{0} ; y-y_{0}, s-s_{0} \mid \underline{r}_{0}, \tau_{0}\right)=G\left(y-y_{0}, s-s_{0} ; x-x_{0}, t-t_{0} \mid-r_{0},-\tau_{0}\right)
$$

where $r_{0}=y_{0}-x_{0}$ and $\tau_{0}=s_{0}-t_{0}$

It is evident that if one integrates (3.3) over all $x$ or all $y$ the one-point Green's function of Sec. 2 is recovered. of more interest is the integral

$$
R\left(\underset{m}{r}, t, s \mid x_{0}, r_{0}, t_{0}, s_{0}\right)=\int d x G\left(x, t ; x+r, s \mid x_{m}, t_{0} ; x_{\infty}+r_{m}, s_{0}\right) .
$$

$R\left(r, t,\left.t\right|_{w 0}, r_{0}, t_{0}, t_{0}\right)$ is Richardson's "distance neighbour function" (Richardson, 1926). It denotes the p.d.f. at time $t$ of separation $r$ for a pair of particles which at time $t_{0}$ were situated at $x_{0}$ and $x_{0}+\underset{0}{r}$ For the homogeneous and stationary flows with which we will be primarily concerned, $R$ depends only on $r_{0}, \tau_{0}, r-r_{0}, t-t_{0}$, and $s-s_{0}$ and will be written

$$
R\left(r-r_{0}, t-t_{0}, s-s_{0} \mid r_{0}, \tau_{0}\right)=\int \operatorname{dxG}\left(x_{0}^{x-x_{0}}, t-t_{0} ;\left(\underset{-x-x_{0}}{x}\right)+\left(\underset{m-r_{0}}{r}\right), s-s_{0} \mid r_{0}, \tau_{0}\right) .
$$


Let us now assume that the Reynolds number of the flow is sufficiently high that an inertial range of wave numbers, or eddy s1zes, exists. By this we mean that the wave numbers which contain most of the energy are distinct from the higher wave numbers which are responsible for most of the energy dissipation. Suppose now, that $r_{0}$ lies with this inertial range of eddy sizes. The eddies of dimension large compared to $r_{0}$ move the two marked particles together bodily without substantially altering the magnltude or direction of $r_{0}$. In a frame of reference moving with these large scale motions, the eddies of dimension small compared to $r_{0}$ are associated with a small r.m.s. velocity and have little effect upon $r_{\text {mono }}$. The rate of separation of the particles, in this case, is dominated by eddies of dimension $r_{0}$, because such edales make the principal contribution to the relative velocity of two points separated by a distance $r_{0}$ (cf. Batchelor, 1953, Ch. 6). These eddies disperse the particles substantially in a time of order

$$
T\left(r_{0}\right)=r_{0}\left[v_{0}^{2}-U_{i i}\left(r_{0}, 0\right)\right]^{-I / 2} \ll \ell / v_{0}
$$

For times short compared to $T\left(r_{0}\right)$, we may apply arguments similar to those of sec. 2. These show that (cf. eq. 2.9)

$$
G\left(x, t ; y, s \mid x_{m}, t_{0} ; y_{0}, s_{0}\right)=\frac{1}{\left(t-t_{0}\right)^{3}\left(s-s_{0}\right)^{3}} P\left(\frac{x-x_{0}}{t-t_{0}}, \frac{y-y_{0}}{s-s_{0}}\right) \text {, }
$$

where $P\left[u_{1}\left(x_{0}, t_{0}\right), u_{2}\left(y_{0}, s_{0}\right)\right]$ is the joint p.d.f. for velocity $u_{1}$ at position $\mathrm{x}_{\mathrm{m}}$ and time $t_{0}$ and velocity $u_{2}$ at position $y_{0}$ and time $s_{0}$ It follows 
(cf.eq. 2.17) that

$$
\begin{aligned}
\left\langle\Delta x_{i} \Delta y_{j}\right\rangle & =\iint \Delta x_{i} \Delta y_{j} G\left(x, t ; y_{m}, s \mid x_{m}, t_{0} ; y_{m}, s_{0}\right) d x d y, \\
& =2 U_{i j}\left(x_{m}, t_{0} ; y_{0}, s_{0}\right)\left(t-t_{0}\right)\left(s-s_{0}\right),
\end{aligned}
$$

where $\Delta x=x-x_{0}$ and $\Delta y=y-y_{0}$. Also, for these short times, (3.7) shows that Richardson's function is

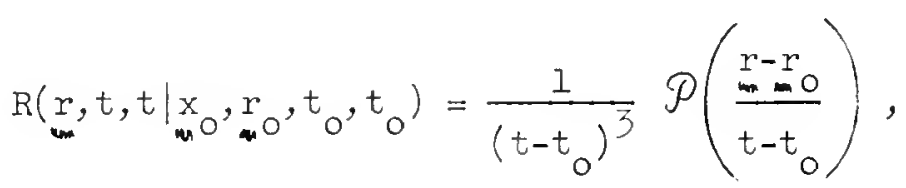

where $\mathcal{P}\left[\mathrm{V}\left(\mathrm{r}_{0}, t_{0}\right)\right]$ is the p.d.f. of relative velocity $\mathrm{V}$ between the two points $x_{m}$ and $x_{m}+\underset{w 0}{r}$ at time $t_{0}$ (cf. Batchelor, 1952a, Sec. 3). It follows from (3.9) that

$$
\left\langle\Delta r_{i} \Delta r_{j}\right\rangle=\left\langle v_{i} v_{j}\right\rangle\left(t-t_{0}\right)^{2}
$$

which, for an isotropic field with $r_{3}$-axis along $\underset{w_{0}}{r}$, gives

$$
\left.\begin{array}{l}
\left\langle\left(\Delta r_{1}\right)^{2}\right\rangle=\left\langle\left(\Delta r_{2}\right)^{2}\right\rangle=2 v_{1}^{2}\left[1-g\left(r_{0}, t_{0}\right)\right]\left(t-t_{0}\right)^{2}, \\
\left\langle\left(\Delta r_{3}\right)^{2}\right\rangle=2 v_{1}^{2}\left[1-f\left(r_{0}, t_{0}\right)\right]\left(t-t_{0}\right)^{2},
\end{array}\right\}
$$

where $f(r, t)$ and $g(r, t)=f(r, t)+\frac{1}{2} r \partial f(r, t) / \partial r$ are respectively the longitudinal and transverse velocity correlations at time $t$ for points separated by a distance $r$. Thus, Richardson's function is initially oblate spheroidal with the line joining the origin $(r=0)$ to $\underset{m}{r}$ as axis. 
In the particular case in which $\mathrm{P}\left(\mathrm{u}_{1}, \mathrm{u}_{2}\right)$ is stationary, isotropic and Gausianly distributed at $x_{0}$ and $y_{w}$, the double-Fourier transform

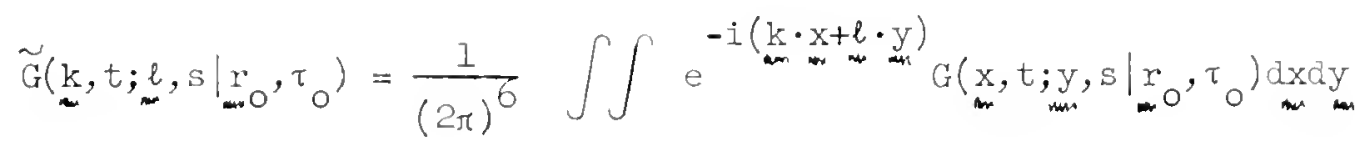

takes the form

$$
\widetilde{G}\left(k, t ; \ell, s \mid x_{m}, \tau_{0}\right)=\frac{1}{(2 \pi)^{6}} \exp \left\{-\frac{1}{2}\left[v_{1}{ }^{2}{ }^{2} t^{2}+v_{1}{ }^{2} \ell^{2} s^{2}+2 U_{1 j}\left(r_{w_{0}}, \tau_{0}\right) k_{i} \ell, t s\right]\right\} .
$$

It follows that Richardson's function

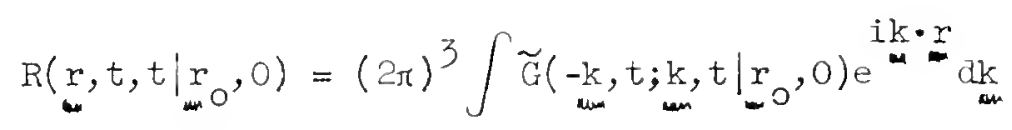

then takes the form

$$
\begin{aligned}
R\left(r, t,\left.t\right|_{m}, 0\right) & =\frac{1}{\pi^{3 / 2}\left(2 v_{1} t\right)^{3}\left[I-g\left(r_{0}\right)\right]\left[1-f\left(r_{0}\right)\right]^{1 / 2}} \\
& \times \exp \left\{-\frac{1}{\left(2 v_{1} t\right)^{2}}\left[\frac{\left(r_{1}^{2}+r_{2}^{2}\right)}{\left[1-g\left(r_{0}\right)\right]}+\frac{r_{3}^{2}}{\left[1-f\left(r_{0}\right)\right]}\right]\right\},
\end{aligned}
$$

where, again, the $r_{3}$-axis is along $r_{\text {mo }}$. 
3.2 Integral equations for $G\left(x, t ; y, s \mid x_{m}, t_{0} ; y_{0}, s_{0}\right)$

When $t-t_{0}$ and $s-s_{0}$ are not small compared to $T\left(r_{0}\right)$, it is no longer legitimate to ignore the spatial and temporal variations of $u_{i}(x, t)$. It is nevertheless possible to effect a partial summation of the formal solution for $G$ containing terms of all orders in the expansion. Since the determination of $G$ is a problem which is essentially inhomogeneous (even if the velocity field is homogeneous), the Fourier modes are not weakly dependent ( are not applicable. However, Kraichnan (1.960) has recently generalized his methods to inhomogeneous problems, and has given an approximate equation of motion for the covariance $\left\langle\psi(\underset{u m}{x}, t) \psi\left(x^{\prime}, t^{\prime}\right)\right\rangle$ of a convected passive scalar field. A straightforward generalization to our case of two scalar fields yields the result

$$
\begin{aligned}
& \frac{\partial}{\partial t} G\left(x, t ; y, s \mid x_{m}, t t_{0} ; y_{0}, s_{0}\right) \\
& =\int_{t}^{t} d t^{\prime} \int d x^{\prime} U_{i j}\left(x, t ; x^{\prime}, t^{\prime}\right) \frac{\partial G\left(x, t \mid x^{\prime}, t^{\prime}\right)}{\partial x_{i}} \frac{\partial G\left(x^{\prime}, t^{\prime} ; y_{m}, s \mid x_{0}, t_{0} ; y_{0}, s_{0}\right)}{\partial x_{j}^{\prime}} \\
& +\int_{s_{0}}^{s} d s^{\prime} \int d y^{\prime} U_{1 j}\left(\underset{m}{x}, t ; y_{m}^{\prime}, s^{\prime}\right) G\left(y_{m}, s \mid y_{m}^{\prime}, s^{\prime}\right) \frac{\partial^{2} G\left(x, t ; y^{\prime}, s^{\prime} \mid x_{0}, t_{0} ; y_{0}, s_{0}\right)}{\partial x_{i} \partial y_{j}^{\prime}},
\end{aligned}
$$

where $G\left(\underset{m_{n}}{x}, t \mid x_{0}, t_{0}\right)$ is the one-point Green's function of Sec. 2. An equation similar to (3.16) holds for $\partial G / \partial s$. The equation for $R\left(\underset{m}{r}, t,\left.s\right|_{m} ^{x}, r_{0}, t_{0}, s_{0}\right)$ that can be obtained from (3.16) by integration may also, in the case of homogeneous velocity fields, be derived quite easily by a device which reduces 
the problem to a fully homogeneous one in which the Fourier moles are weakly dependent. This matter is dealt with briefly in Appendix B. For velocity fields which are homogeneous and stationary, (3.16) may be written, after partial integration, in the form

$$
\begin{aligned}
& \frac{\partial G\left(x, t ; y, s \mid r_{m 0}, \tau_{0}\right)}{\partial t}=\frac{\partial^{2}}{\partial x_{i} \partial x_{j}} \int_{0}^{t} d t^{\prime} \int d x^{\prime} U_{i j}\left(x_{m}^{\prime}, t^{\prime}\right) G\left(x_{m}^{\prime}, t^{\prime}\right) G\left(x-x^{\prime}, t-t^{\prime} ; y_{m}, s \mid r_{0}, \tau_{0}\right)
\end{aligned}
$$

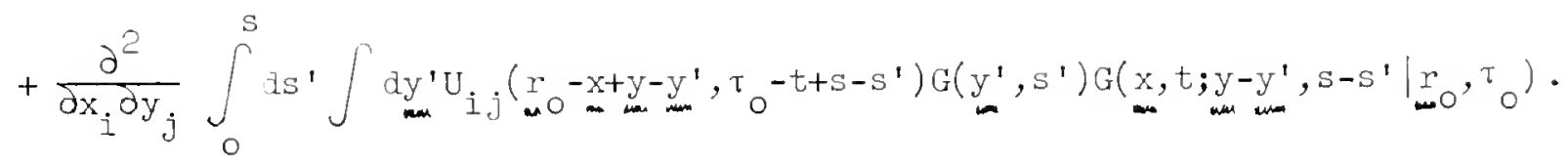

The equation for $R\left(\underline{r}, t, s||_{n+0}, \tau_{0}\right)$ to which we referred above is

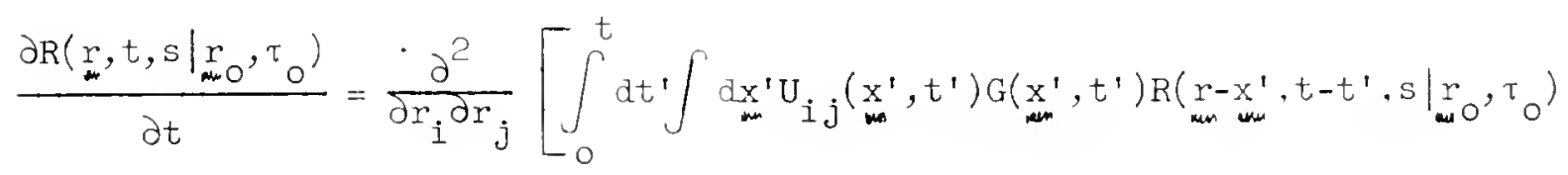

$$
\begin{aligned}
& \left.-\int_{0}^{S} d s^{\prime} \int d y^{\prime} U_{i j}\left(r_{0}^{r}+r-y_{m}^{\prime}, \tau_{0}+\tau-s^{\prime}\right) G\left(y_{m}^{\prime}, s^{\prime}\right) R\left(\underset{m}{r-y_{m}^{\prime}}, t, s_{m}-\left.s^{\prime}\right|_{m 0^{\prime}}, \tau_{0}\right)\right] .
\end{aligned}
$$

On multiplying this equation by $r_{i} r_{j}$ and integrating by parts, we find

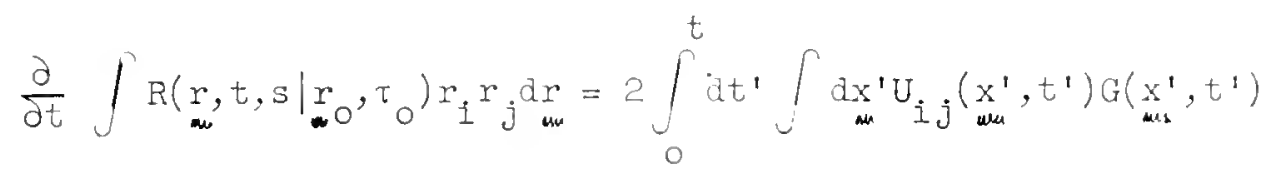

$$
\begin{aligned}
& -2 \int_{0}^{s} d s^{\prime} \int \operatorname{drU} i j\left(\underset{\mu O}{r}+r, \tau_{0}+s^{\prime}-t\right) R\left(\underset{m}{r}, t, s^{\prime}||_{m}^{r}, \tau_{0}\right) .
\end{aligned}
$$


By differentiating (3.19) with respect to $\mathrm{s}$, we find

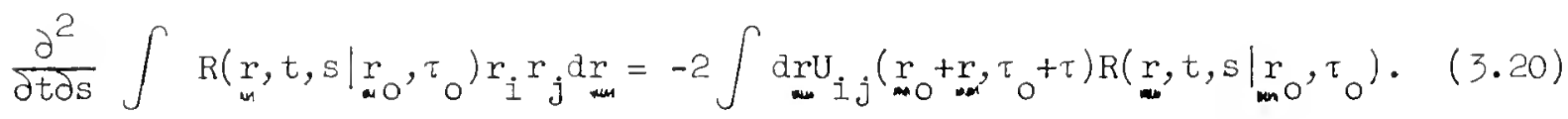

Let us take $t_{0}=0, s_{0}=0$. Then, by (3.20) and (2.32), we see that the Lagrangian correlation for relative velocity $V$ between two particles which were initially separated by a distance $r$ is

$$
\left\langle V_{i}(t) V_{j}(s)\right\rangle_{L}=2 \int \operatorname{ard}_{i j}(\underset{m, s}{r}, t)[G(\underset{m}{r}, s-t)-R(\underset{m}{r-r}, t, s \mid \underset{m o}{r}, 0)] .
$$

This result should be compared to the exact result

$$
\begin{aligned}
\left\langle v_{1}(t) v_{j}(s)\right\rangle_{L}=\iint d x d y & \left\langle\psi_{1}(x, t) \psi_{1}(y, s) u_{i}(x, t) u_{j}(y, s)\right\rangle \\
& +\left\langle\psi_{2}(x, t) \psi_{2}(y, s) u_{i}(x, t) u_{j}(y, s)\right\rangle \\
& -\left\langle\psi_{1}(x, t) \psi_{2}(y, s) u_{i}(x, t) u_{j}(y, s)\right\rangle \\
& \left.-\left\langle\psi_{2}(x, t) \psi_{1}(y, s) u_{i}(x, t) u_{j}(y, s)\right\rangle\right],
\end{aligned}
$$

where $\psi_{1}(x, t)=\psi_{m}\left(x_{m},\left.t\right|_{m}, 0\right)$ and $\psi_{2}(y, s)=\psi_{2}\left(y,\left.s\right|_{m} y_{m}, 0\right)$ denote the unaveraged Green's functions. In stationary homogeneous flows, this result reduces to (3.21) on the assumption that the $\psi$ and $u$ fields are uncorrelated (cf. Sec. 2.3) 
Also, by alding to (3.19) to the analagous equation for the derivative with respect to $s$, and setting $t=s$, we find

$$
\begin{aligned}
& \frac{\partial}{\partial t}\left\langle r_{i} r_{j}\right\rangle=\int \frac{\partial}{\partial t} R\left(\underline{r}, t,\left.t\right|_{r} ^{r}, 0\right) r_{i} r_{j} d r= \\
& =4 \int_{0}^{t} d t^{\prime} \iint_{m}^{\prime} U_{1 j}\left(\underset{\mu m}{x^{\prime}}, t^{\prime}\right) G\left(x_{n+m}^{\prime}, t^{\prime}\right)
\end{aligned}
$$

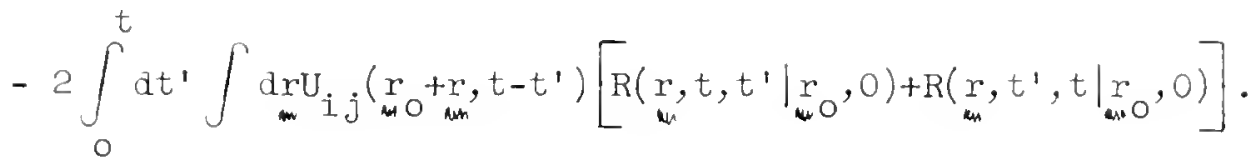

For $t \ll T\left(r_{0}\right), R$ is negligible except near $\underset{m}{r}=0$, and the right-hand side of (3.22) becomes

$$
4\left[U_{i j}(0,0)-U_{i j}\left(r_{m}, 0\right)\right] t,
$$

in agreement with (3.10). For $t \gg \ell / v_{0}, R$ is appreciable even at for separations $r$ of order $\ell$. For such large separations, the second term on the right-hand side of (3.22) is quite negligible, and we find (cf. eqs. 2.27, 2.28)

$$
\left\langle r_{i} r_{j}\right\rangle=2\left\langle x_{i} x_{j}\right\rangle=2\left\langle y_{i} y_{j}\right\rangle=4 k_{i j} \cdot
$$

This is consistent with the intuitive notion that, at such large separations from their source and each other, the particles will wander independently as 
28.

in a tme Brownian motion. For intermediate ranges of $t$, it appears that no such definite statements can be made. However a reasonable approximation appears to be possible and this is discussed in sec. 3.4 .

\subsection{Solution of equations for short times}

For $t \ll T\left(r_{0}\right)$, we may assume that the $G$ factors in the integrands of (3.17) are both negligible unless $x^{\prime}, y_{m}^{\prime}, x_{m}, y_{m}$ are small compared with $r_{m} \cdot$. Then, the $U_{i j}$ factors in the integrands may be replaced by their values for zero $\mathrm{x}^{\prime}, \mathrm{y}^{\prime}, \mathrm{x}, \mathrm{y}$, and similarly for the time arguments. Therefore, in isotropic flows, we have

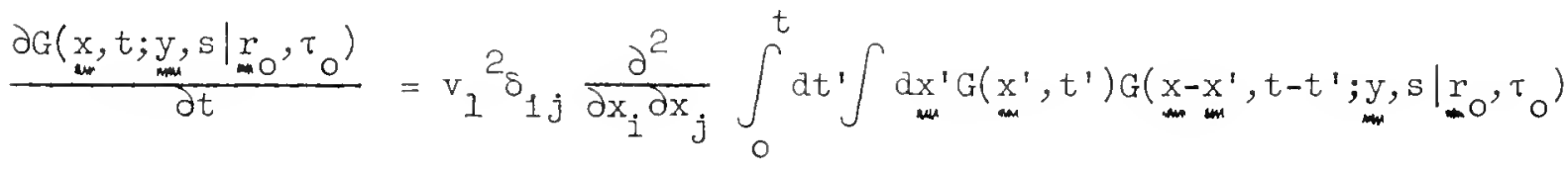

$$
\begin{aligned}
& -U_{i j}\left(r_{\infty}, \tau_{0}\right) \frac{\partial^{2}}{\partial x_{i} \partial y_{j}} \int_{0}^{S} d s^{\prime} \int d y^{\prime} G\left(y_{m}^{\prime}, s^{\prime}\right) G\left(x, t ; y-y^{\prime}, s_{m}-s^{\prime} \mid r_{m}, \tau_{0}\right) .
\end{aligned}
$$

Take a combined Laplace and Fourier transform defined by

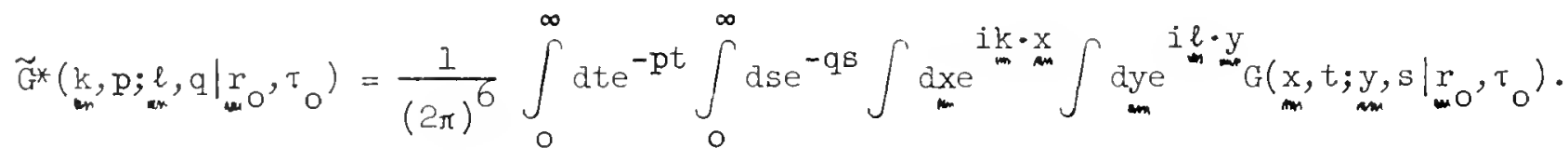

Then, by $(3.24)$, 


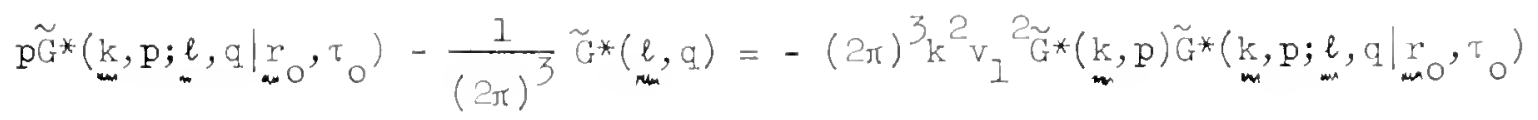

$$
\begin{aligned}
& -(2 \pi)^{3} U_{i j}\left(r_{0}, \tau_{0}\right) k_{i} \ell, \widetilde{G}^{*}(\ell, q) \tilde{G}^{*}\left(k, p ; \ell,\left.q\right|_{m} r_{m}, \tau_{0}\right),
\end{aligned}
$$

where $\widetilde{G}^{*}(\mathrm{k}, \mathrm{p})$ is the Fourjer transform with respect to $\mathrm{x}$ and the Laplace transform with respect to $t$ of $G(x, t)$. By $(2 \cdot 34)$, we have

$$
p+(2 \pi)^{3} k^{2} v_{I}^{2} \tilde{G}^{*}(k, p)=\frac{I}{(2 \pi)^{3} \tilde{G}^{*}(\underset{m, p}{k}, p)}
$$

By $(3.26)$ and $(3.27)$, it follows that.

$\widetilde{G}^{*}\left(\underset{m}{k}, p ; \ell, q \mid \underset{m}{r_{0}}, \tau_{0}\right)=\widetilde{G}^{*}(k, p) \tilde{G}^{*}(\ell, q) /[I+(2 \pi) U_{i j}\left(\underset{m 0}{r}, \tau_{0}\right) k_{i} \underbrace{}_{j} \widetilde{G}^{*}(k, p) \widetilde{G}_{m}^{*}(\ell, s)]$.

If we expand the denominator of (3.28), we obtain

$\tilde{G}^{*}\left(k_{m}, p ; \ell, q \mid r_{m}, \tau_{0}\right)=\sum_{0}^{\infty}(-I)^{m}\left[(2 \pi)^{\sigma} U_{i j}(\underbrace{r}_{m}, \tau_{0}) k_{i} \ell\right]^{m}\left[\tilde{G}^{*}(k, p) \tilde{G}_{m}^{*}(\ell, q)\right]^{m+I}$.

Now, by $(3.27)$

$$
\tilde{G}^{*}(\underset{m}{k}, p)=I /\left\{4 \pi^{3}\left[p+\sqrt{p^{2}+4 k^{2} v_{1}^{2}}\right]\right\} .
$$


The inverse Laplace transformation of $\left[\tilde{G}^{*}(k, p)\right]^{m+l}$ is therefore (see, for example, Watson, 1944, Sec. 13.2, eq. 7)

$$
\frac{(m+1) J_{m+1}\left(2 k v_{1} t\right)}{(2 \pi)^{3 m+3}\left(k v_{1}\right)^{m+I_{t}}}
$$

Thus

$$
\begin{gathered}
\tilde{G}\left(k, t ; \ell, s \mid r_{m}, \tau_{0}\right)=\frac{1}{(2 \pi)^{6}} \sum_{0}^{\infty}(-I)^{m}(m+1)^{2}\left[\frac{k_{i}^{\ell} U_{i j}\left(r_{0}, \tau_{0}\right)}{k v_{I}^{2}}\right]^{m} \\
\times \frac{J_{m+1}\left(2 k v_{I} t\right)}{k v_{I} t} \frac{J_{m+I}\left(2 \ell v_{i} s\right)}{\ell v_{I} s} .
\end{gathered}
$$

The second moments of (3.31) agree with (3.8); for a normally distributed velocity distribution, the higher moments are given with progressively less accuracy (cf. Sec. 2.4). In Appendix B, the significance of (3.31) in terms of diagrams is discussed. The relationship

$$
\int \frac{J_{m+1}\left(2 k v_{I} t\right)}{\left(k v_{I} t\right)^{m+1}} e^{i k \cdot x} d x= \begin{cases}\frac{2 \pi m !}{(2 m) !} \frac{\left(4 v_{I}^{2} t^{2}-x^{2}\right)^{m-1 / 2}}{\left(v_{I} t\right)^{2 m+2}} & , \text { if } x<2 v_{1} t \\ 0 & , \text { if } x>2 v_{1} t\end{cases}
$$

can be obtained by induction from results of Watson (1944, Sec. 13.42, eq. 4, $\mu=1$, using also Sec. 13.24, eq. I) or directly (Watson, Sec. 13.14). This enables us to invert the function $\widetilde{G}\left(k, t ; \ell, s \mid r_{w 0}, \tau_{0}\right)$ in the form (cf. eq. 2.36) 


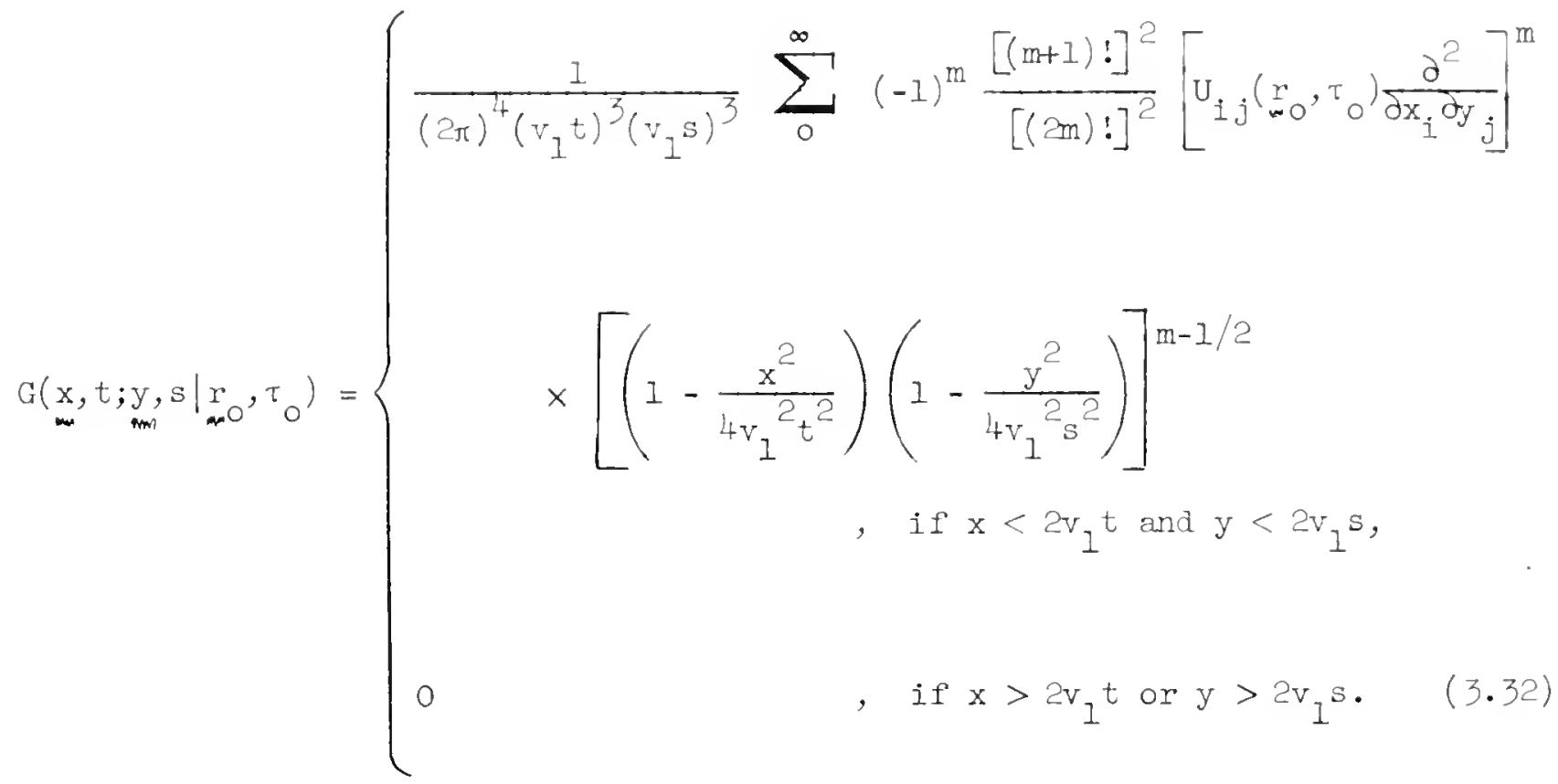

As in sec. 2.3, the effect of the artificial cut-off $2 v_{1}$ in the p.d.f. of velocity is apparent. It is also clear that, if $U_{i j}\left(r_{0}, \tau_{0}\right)$ is small compared to $v_{1}^{2},(3.32)$ reduces to its first term

$$
G\left(\underset{m}{x}, t ; y, s \mid x_{m}, \tau_{0}\right) \doteq G(x, t) G(y, s),
$$

where the right-hand side is given by (2.36). If the initial separation of the points is so close in space and time that we can write $U_{i j}\left(r_{0}, \tau_{0}\right)=v_{i}{ }^{2} \delta_{i j}$, equations (3.14) and (3.31) show (cf. Watson Sec. 11.41, eq. (12)) that

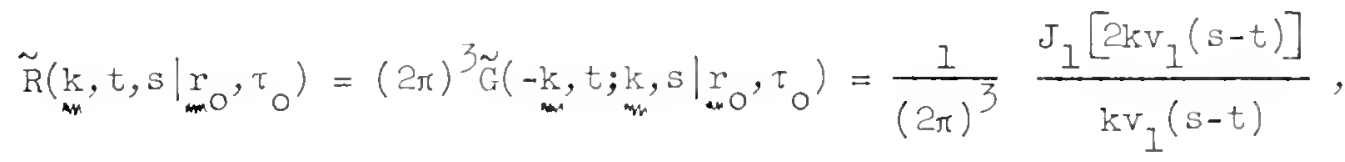

or, inverting, 


$$
R\left(\underset{s}{r}, t,\left.s\right|_{m, 0}, \tau_{0}\right) \rightarrow G\left(r_{0}^{r}+r, s-t\right) ; \underset{r+0}{r} \rightarrow 0, \tau_{0} \rightarrow 0
$$

This shows, as we expect, that in the limit $\underline{r}_{0} \rightarrow 0, \tau_{0} \rightarrow 0$, the particles are not separated by the flow and simply move together as one particle. (As a further consistency check, we note that the Lagrangian correlation (3.21) is given correctly by (3.33) in this case.)

\subsection{Solutions for large and intermediate times}

For very long times, $t \gg \ell / v_{0}$, there is a high probability that the particles have separated by a distance comparable to, or greater than, $\ell$ and therefore wander independently. In fact, the solution of (3.17) is exactly analagous to that of Sec. 2.5 for the one-point Green's function, and we find

$$
G\left(x, t ; \underset{m}{y},\left.s\right|_{m, 0} ^{r}, \tau_{0}\right) \longrightarrow G(\underset{w}{x}, t) G(\underset{w i}{y}, s), \quad(r>>l),
$$

where $G(x, t)$ and $G(y, s)$ are given, in homogeneous flows, by the appropriate solutions of $(2.45)$. In this case we find $R(\underset{r}{r}, t)$ satisfies

$$
\frac{\partial R(\underset{m,}{r}, t)}{\partial t}=2 k_{i j} \frac{\partial^{2} R(\underset{m}{r}, t)}{\partial r_{i} \partial r_{j}},
$$

so that $R(r, t)$ assumes a Gaussian form corresponding to a diffusivity twice that characteristic of the one particle Green's function (cf. eqs. 2.45, 3.23). Consider turbulence at high Reynolds numbers. The existence of an inertial range imples that the spectrum $\mathrm{E}(\mathrm{k})$ obeys 


$$
\begin{aligned}
& \int_{0}^{\mathrm{k}} \mathrm{E}(\mathrm{k}) \mathrm{dk} \doteq \frac{1}{2} \mathrm{v}_{0}^{2}, \quad \text { if } \mathrm{k} \gg \mathrm{k}_{0} \text {, } \\
& \int_{k}^{\infty} E(k) k^{2} d k \doteq \frac{1}{2} \epsilon / v, \text { if } k \ll k_{a} \text {, }
\end{aligned}
$$

where $k_{0}=(l / \ell)$ and $k_{d}$ are wave numbers characteristic of the energy containing range and the dissipation range, respectively. ( $\epsilon$ = rate of dissipation per unit mass; $v=$ kinematical viscosity.) It follows that

$$
\mathrm{kE}(\mathrm{k}) \rightarrow 0, \mathrm{k} \rightarrow \infty ; \mathrm{k}^{3} \mathrm{E}(\mathrm{k}) \rightarrow 0, \mathrm{k} \rightarrow 0
$$

For the later developments ( $\mathrm{cf}$. eqs. 3.53, 3.54 below), we will require the more stringent conditions

$$
k E(k) \rightarrow 0, k \rightarrow \infty ; k^{2} E(k) \rightarrow 0, k \rightarrow 0
$$

If the intial separation satisfies

$$
\ell \gg r_{0} \gg 1 / k_{d}
$$

we expect that, in a time large compared to $T\left(r_{0}\right)$ but small compared to $\ell / v_{0},\left\langle\left(r-r_{0}\right)^{2}\right\rangle$ will become large compared to $r_{0}^{2}$ but remain small compared to $\ell^{2}$. For these "Intermediate times" (as we shall term them), neither the short-time solution of Sec. 3.3 nor the long-time solution above is valid. 
Consider $R\left(r-r, t, t+\left.\tau\right|_{m}, 0\right)$ for $\tau>0$. This quantity is the p.d.f. of the separation of two particles (released at $t=0$ at a separation of $r_{0}$ ) one of which has been carried by the flow for a time $t$, and the other for a time t+t. This process may be visualised in two stages. During the first, of duration $t$, both particles are carried by the flow. Their separation $r$ during this time is essentially unaffected by the energy-containing eddies which give nearly equal displacements to both particles. It is governed by the motions (relative to the energy containing eddies) of dimension $\sim r$. Now, in a frame moving with the energy-containing eddies, the r.m.s. velocity associated with these small scale motions is very small compared to $\mathrm{v}_{0}$. Thus, the mean square separation $\left\langle r^{2}\right\rangle$ at the termination of the first stage: is very small compared to $\left(v_{0} t\right)^{2}$. During the second stage, of duration $\tau$, one of the particles can be considered as fixed in space while the other is carried by the flow for a further time $\tau$. Its motion during this time is dominated by the energy-containing motions. During the first stage the relative diffusion is given by $R\left(r, t, t||_{w 0}^{r}, 0\right)$. During the second stage the further diffusion of the second particle should be given by $G(r, \tau)$, since the energy-containing motions are almost uncorrelated with the small scale motions. Thus, we expect

$$
R\left(r, t, t+\tau||_{m}, 0\right) \doteq \int R\left(\underset{m}{r^{\prime}}, t, t \mid \underset{m}{r}, 0\right) G\left(\underset{m}{r-r_{m}^{\prime}}, \tau\right) d r^{\prime}
$$

It is clear that this result is exact for $t=0$ or $\tau=0$. Also, the change in the mean square separation during the second stage will be of the order of $\left(v_{0} \tau\right)^{2}$. Thus, when this is large compared to the value of $\left\langle r^{2}\right\rangle$ at the end of the first stage, (3.37) becomes 


$$
R(r, t, t+\tau \mid r, 0) \doteq G(r, \tau)
$$

Consider now (3.18) and its counterpart for $\partial R / \partial s$. These show that

$$
\begin{aligned}
& \frac{\partial R\left(r, t, t \mid r_{m}, 0\right)}{\partial t}=\frac{\partial^{2}}{\partial r_{i} \partial r_{j}}\left\{\int_{0}^{t} d t^{\prime} \int d r_{m}^{\prime}\left[U_{i j}\left(r_{m}^{\prime}, t^{\prime}\right)-U_{i j}\left(r_{0}+r-r^{\prime}, t^{\prime}\right)\right]\right. \\
& \left.\times G\left(r_{m}^{\prime}, t^{\prime}\right)\left[R\left(r-r_{m}^{\prime}, t-t^{\prime},\left.t\right|_{m} ^{r}, 0\right)+R\left(r-r_{m}^{\prime}, t, t-t^{\prime} \mid r, 0\right)\right]\right\} .
\end{aligned}
$$

By the arguments above, the integrand should be dominated by contributions from small t' (because of the behavior of $R$ ) and from small $r^{\prime}$ (because of the behavior of $G$ ). We will therefore replace the $R$ factors in the integrand by $R\left(r, t,\left.t\right|_{m}, 0\right)$. It seems likely from the preceding discussion that this approximation will lead to results which are at least qualitatively correct.

We now find

$$
\frac{\partial R\left(r, t,\left.t\right|_{m}, 0\right)}{\partial t}=\frac{\partial^{2}}{\partial r_{i} \partial r_{j}}\left[K_{i j}(\underset{m}{r}, t) R\left(\underset{m}{r}, t,\left.t\right|_{m} ^{r}, 0\right)\right]
$$

where

$$
K_{i j}(r, t)=2 \int_{0}^{t} d t^{\prime} \int d r_{m}^{\prime}\left[U_{i j}\left(r_{m}^{\prime}, t^{\prime}\right)-U_{i j}\left(\underset{m}{r}+r-r_{m}^{\prime}, t^{\prime}\right)\right] G\left(r_{m}^{\prime}, t^{\prime}\right) .
$$

For short times $t \ll T\left(r_{0}\right),(3.41)$ becomes 


$$
K_{i j}(r, t)=2 t\left[v_{1}^{2} \delta_{i j}-U_{i j}(r, 0)\right]
$$

which, by (3.40), is in agreement with (3.10). For very large times $t \gg \ell / v_{0}$, there is a high probability that the separation of particles is $\geqslant \ell$. For these separations, the second $U$ factor in the integrand of (3.4I) is negligible, and the first factor gives

$$
K_{i j}(r, t)=2 \kappa_{i j},
$$

in agreement with the results derived earlier (cf. 3.23).

To calculate the form of $\mathrm{K}_{i j}(\mathrm{r}, \mathrm{t})$ for intermediate times, we express (3.41) In the form

$K_{i j}(\underline{r}, t)=2(2 \pi)^{3} \int_{0}^{t} d t \cdot \int d k\left[1-e^{i k \cdot\left(\underset{m}{r}+\underline{r}_{0}\right)}\right] \tilde{U}_{i j}\left(\underset{m}{k}, t^{\prime}\right) \widetilde{G}\left(k, t^{\prime}\right)$.

Assuming 1sotropy, we can write

$$
\tilde{U}_{i j}(k, t)=\left(\delta_{i j}-\frac{k_{i} k j}{k^{2}}\right) \frac{E(k, t)}{4 \pi k^{2}}
$$

where $E(k, 0)$ is the energy spectrum. It follows that if we adopt spherical polar coordinates and write

$$
R(\underbrace{r-r}_{m 0}, t, t||_{m 0}^{r}, 0)=R(r, \theta, \phi, t)
$$

(3.40) has the form

$\frac{\partial R}{\partial t}=\frac{1}{r^{2}} \frac{\partial}{\partial r}\left[r^{2} K(r, t) \frac{\partial R}{\partial r}\right]+\frac{1}{r^{2}} M(r, t)\left[\frac{1}{\sin \theta} \frac{\partial}{\partial \theta}\left(\sin \theta \frac{\partial R}{\partial \theta}\right)+\frac{1}{\sin ^{2} \theta} \frac{\partial^{2} R}{\partial \phi^{2}}\right]$, 
where, by $(3.44)$ and $(3.45)$

$K(r, t)=4(2 \pi)^{3} \int_{0}^{t} d t^{\prime} \int_{0}^{\infty} d k\left[\frac{1}{3}-\frac{\sin k r}{(k r)^{3}}+\frac{\cos k r}{(k r)^{2}}\right] E\left(k, t^{\prime}\right) \tilde{G}\left(k, t^{\prime}\right)$,

$M(r, t)=2(2 \pi)^{3} \int_{0}^{t} d t^{\prime} \int_{0}^{\infty} d k\left[\frac{2}{3}-\frac{\sin k r}{k r}+\frac{\sin k r}{(k r)^{3}}-\frac{\cos k r}{(k r)^{2}}\right] E\left(k, t^{\prime}\right) \tilde{G}\left(k, t^{\prime}\right)$.

For small kr, the quantities in the square brackets $[\ldots .$.$] in the$ integrands of (3.47) and (3.48) are proportional to $(\mathrm{kr})^{2}$. Also, in the inertial range, we may take (cf. eq. 2.21)

$$
\tilde{G}(k, t)=\frac{1}{(2 \pi)^{3}} \exp \left[-\frac{1}{2} k^{2} v_{1}^{2} t^{2}\right]
$$

and (cf. Kraichnan, 1959, Sec. 8.4)

$$
E(k, t)=E(k) \exp \left[-\frac{1}{2} k^{2} v_{1}^{2} t^{2}\right]
$$

Thus, since by our initial supposition (3.35), $\mathrm{k}^{2} \mathrm{E}(\mathrm{k}) \rightarrow 0, \mathrm{k} \rightarrow 0$, it follows that the integrands of (3.47) and (3.48) do not tend to infinity as rapidly as $k^{-1}, k \rightarrow 0$. Consequently, the form of $E(k)$ for small $k\left(\sim k_{0}\right)$ does not influence the values of $K(r, t)$ and $M(r, t)$ appreclably. However, these quantities do depend implicitly on the energy containing range through the forms (3.49) and (3.50) for $\tilde{G}(k, t)$ and $E(k, t)$. We will discuss this in more detail at the conclusion of this section. 
Three further approximations are clearly justified. First since $\left\langle\left(r-r_{0}\right)^{2}\right\rangle$ is large compared to $r_{0}^{2}$ for the times under consideration, $r_{0}$ may be neglected in (3.47) and (3.48). Clearly, also, the particles have in this time lost all memory of the initial orientation of $r$, so that $R(r, \theta, \phi, t)$ is spherically symmetrical and, by (3.46), satisfies

$$
\frac{\partial R(r, t)}{\partial t}=\frac{1}{r^{2}} \frac{\partial}{\partial r}\left[r^{2} K(r, t) \frac{\partial R(r, t)}{\partial r}\right] .
$$

Lastly, since $\left\langle r^{2}\right\rangle$ is small compared to $\left(v_{0} t\right)^{2}$, the exponential factors of (3.49) and (3.50) are small at the upper limt of integration over $t$ in (3.47) and (3.48), and we may therefore wxite

$$
\begin{aligned}
K(r, t) & =K(r)=4 \int_{0}^{\infty} d t \int_{0}^{\infty} d k\left[\frac{1}{3}-\frac{\sin k r}{(k r)^{3}}+\frac{\cos k r}{(k r)^{2}}\right] E(k) \exp \left[-\frac{1}{3} k^{2} v_{0}^{2} t^{2}\right] \\
& =2 \sqrt{3 \pi} \int_{0}^{\infty} \frac{d k}{v_{0} k}\left[\frac{1}{3}-\frac{\sin k r}{(k r)^{3}}+\frac{\cos k r}{(k r)^{2}}\right] E(k) .
\end{aligned}
$$

Equation (3.51) was proposed by Richardson (1926) for the 1ntermediate times discussed here. To investigate further the form of the variable diffusion coefficient (3.52), we will assume that in the inertial range the spectrum is a power law ${ }^{*}$ of the form (cf. eq. (3.35)

We may now take the wave number $k_{d}$ characteristic of the dissipation range to be

$$
\mathrm{k}_{\mathrm{d}}=\mathrm{R}_{\mathrm{o}}^{\mathrm{l} /(3-\mathrm{n})} \mathrm{k}_{\mathrm{o}}
$$

(This footnote continued on p. 39) 


$$
E(k)=\beta \epsilon^{n-1} v_{0}^{5-3 n} k^{-n}, \quad(I<n<2),
$$

where $\beta$ is a dimensionless constant of the order of unity. W1th this spectrum, (3.52) gives on integrating by parts and use of results of Watson (1944, Secs. $3.4,13.24)$,

$$
\begin{aligned}
K(r) & =\frac{\beta \pi \sqrt{6}}{n} \epsilon^{n-1} v_{0}^{4-3 n} r^{n} \int_{0}^{\infty} \frac{J_{5 / 2}(x) d x}{x^{n+3 / 2}} \\
& =\frac{\beta \pi \sqrt{3}}{2^{n+1} n} \frac{\Gamma\left(1-\frac{1}{2} n\right)}{\Gamma\left(\frac{5}{2}+\frac{1}{2} n\right)} \epsilon^{n-1} v_{0}^{4-3 n} r^{n} \equiv \lambda r^{n}, \text { (say). }
\end{aligned}
$$

(Continuation of footnote on page 38.)

where

$$
\mathrm{k}_{0} \equiv \mathrm{l} / \mathrm{e}=\epsilon / \mathrm{v}_{0}^{3}, \mathrm{R}_{0}=\mathrm{v}_{0}^{4} / \epsilon v=\mathrm{v}_{0} / \mathrm{k}_{0} v
$$

The "intermediate times" referred to above may now be defined more precisely by

$$
T\left(r_{0}\right)=\left(\frac{r_{0}}{l}\right)^{(3-n) / 2} \frac{\ell}{v_{0}} \ll t \ll \frac{l}{v_{0}} .
$$

Also, the circumstances under which (3.38) is a good approximation are

$$
v_{0} \tau / \ell>\left(v_{0} t / \ell\right)^{1 /(2-n)}
$$

Note that (3.53) may be written

$$
E(k)=\beta \ell v_{0}^{2}(k l)^{-n}
$$


If we suppose that at some time $t_{1} \gg T\left(r_{0}\right)$

$$
R(r, t)=R\left(r, t_{1}\right)
$$

then at subsequent times $t\left(\ll \ell / v_{0}\right),(3.51)$ and $(3.54)$ show that

$R(r, t)=\frac{1}{2}(2-n) r^{-\frac{1}{2}(n+1)} \int_{0}^{\infty} \widetilde{\mathcal{R}}\left(\xi, t_{1}\right) J_{S}\left(\xi r^{\frac{1}{2}(2-n)}\right) e^{-\frac{1}{T^{2}} \lambda(2-n)^{2} \xi^{2}\left(t-t_{1}\right)} \xi d \xi$,

where $s=(1+n) /(2-n)$ and

$$
\tilde{R}\left(\xi, t_{1}\right)=\int_{0}^{\infty} r^{\frac{1}{2}(3-r)} \partial(r, t) J_{s}\left(\xi r^{\frac{1}{2}(2-r)}\right) d r .
$$

For $t \gg t_{1},(3.56)$ reduces to ${ }^{*}$

$R(r, t)=\frac{1}{(2-n)^{4+n / 2-n} \Gamma\left(\frac{3}{2-n}\right)} \cdot \frac{1}{(\lambda t)^{\frac{3}{2-n}}} e^{-r^{2-n} /(2-n)^{2} \lambda t}$,

so that

$$
\left\langle r^{2}\right\rangle=(2-n)^{4 / 2-n} \frac{\Gamma\left(\frac{5}{2-n}\right)}{\Gamma\left(\frac{3}{2-n}\right)}(\lambda t)^{2 / 2-n}
$$

The behàvior of $R(r, t)$ and $\left\langle r^{2}\right\rangle$ given by (3.58) and (3.59) is almost certainly independent of the approximation

$$
R\left(\underline{r}_{m}^{r} \underline{r}^{\prime}, t-t^{\prime},\left.t\right|_{m} ^{r}, 0\right) \doteq R\left(r_{m}^{r}, t,\left.t\right|_{m} ^{r}, 0\right)
$$

which led from (3.39) to (3.40). In fact, it can be shown that the more accurate approximation method based on (3.37) and (3.39) leads to results whose dimensional forms are identical to (3.58) and (3.59). However, it is worth confirming a posterlori that the approximation we have adopted is good. 
The behavior of $\left\langle r^{2}\right\rangle$ as a function of $t$ is extraordinarily sensitive to the value of $n$ assumed. Two cases are worthy of notice:

$$
\begin{aligned}
& n=3 / 2(\text { Kraichnan, 1959) } \\
& \mathrm{K}(\mathrm{r}) \propto \epsilon^{1 / 2} \mathrm{r}^{3 / 2 / v_{0} 1 / 2},\left\langle\mathrm{r}^{2}\right\rangle \propto \epsilon^{2} \mathrm{t}^{4 / v_{0}^{2}}, \\
& \mathrm{n}=5 / 3(\text { Kolmogrov, 1941) } \\
& \mathrm{K}(\mathrm{r}) \propto \epsilon^{2 / 3} \mathrm{r}^{5 / 3 / v_{0},}\left\langle\mathrm{r}^{2}\right\rangle \propto \epsilon^{4} \mathrm{t}^{6 / v_{0}^{6}} .
\end{aligned}
$$

Neither of these agree with the form proposed by Richardson (1926, see also Batchelor, 1950):

$$
K(r) \propto \epsilon^{1 / 3} r^{4 / 3},\left\langle r^{2}\right\rangle \propto \in t^{3}
$$

That this is so is not surprising. Kraichnan's direct interaction approximation does not give an inertial range spectrum which agrees with that derived from Kolmogorov's similarity arguments. In the same way, when this approximation is applied to turbulent diffusion, it does not give a diffusion coef-

(Continuation of footnote on p. 40.)

For this purpose, we expand $R\left(\underline{r}-r_{m}^{\prime}, t-t^{\prime},\left.t\right|_{w_{0}} ^{r}, 0\right)$ in a serles about $\underline{r}^{\prime}=0$, $t^{\prime}=0$; substitute in (3.39); and, using (3.58), compare the second term of the series with the first (cf. similar argument of sec. 2.5). We find that this second term is smaller than the first by a factor of the order of $\left(r / v_{0} t\right)$ so that, for $t \ll \ell / v_{0}$ (at which $t$ imes $\left\langle r^{2}\right\rangle$ is small compared to $\left.\ell^{2}\right)$, we may expect $(3.58)$ to be a good approximation to $R(r, t)$. 
ficient which agrees w1th that derived by these similarity arguments, even if Kolmogorov's spectrum is assumed. This is because the dynamics of diffusion with a given velocity field differ on the two theories. It would seem that the behavior of $\left\langle x^{2}\right\rangle$ as a function of $t$ might provide a sensitive test by which to confront with experiment different assumptions about the structure of the inertial range.

Kraichnan (1959) has made a detailed comparison between the directinteraction approximation and the Kolmogorov theory. He has traced the difference in the inertial range spectra to the difference in the role played by the energy-containing eddies in the two cases. In Kolmogorov's theory these edales merely convect the small scale motions without influencing their dynamics, whereas in the direct-interaction approximation this is not so. In the same way, on arguments of the Kolmogorov type, the relative diffusion of particles should be independent of the energycontaining edaies. However, the application of the direct-interaction approximation has led to results which depend on the energy-containing motions. If we wish to modify our formalism in such a way that our results depend only on the small scale motions, we would transform to a frame of reference moving with the energy-containing eddies. We would expect that results of the form $(3.40),(3.41)$ would be qualitatively correct, provided that $\tilde{G}(k, t)$ now described one-point diffusion relative to a source moving with the energy-containing edaies. On similarity arguments of the Kolmogorov type, $\widetilde{G}(k, t)$ would then depend only upon $k^{2 / 3} \epsilon^{1 / 3} t$. Similarly $\mathrm{E}(\mathrm{k}, \mathrm{t})$ would describe the structure of the small-scale eddies in a frame of reference moving with the large scale motions, and would take the form 


$$
E(k, t)=E(k) f\left(k^{2 / 3} \epsilon^{1 / 3} t\right)
$$

The overall effect of these modifications would be that $[\mathrm{kE}(\mathrm{k})]^{1 / 2}$ would appear in place of $v_{0}$ in the expression (3.52) for $K(r)$. Kraichnan (1959, sec. 9.1) has shown that this substitution resolves the conflict between the Kolmogorov theory of turbulence and the theory based on the direct-interaction approximation. The quantity $[\mathrm{k} E(\mathrm{k})]^{1 / 2}$ may be considered as the $r \cdot m \cdot s$. velocity associated with the motions of wave numbers $k$ as they are convected by the large-scale motions. Substitution of this quantity for $v_{0}$ in (3.52) gives

$$
K(r) \propto \epsilon^{\frac{1}{2}(n-1)} v_{0}^{\frac{1}{2}(5-3 n)} \frac{1}{r^{2}(n+1)},
$$

which, taking $n=5 / 3$, leads to (3.62). With these changes in the interpretation of $\widetilde{G}(k, t)$ and $E(k, t)$, the sensitivity of our results to the form of the inertial range spectrum remains.

\section{Acknowledgement}

I am extremely grateful to Dr. R.H. Kralchnan for detalled discussions and helpful criticisms of the work presented in this paper. 


\section{4.}

\section{APPENDIX A:}

\section{Derivation of equation (2.26)}

When the velocity field is spatially homogeneous, the problern of diffusior from a point source, although apparently possessing only radial synmetry even in the isotropic case, can always be cepinrased as a homogeneous problem. For, since the equation (2.2) is linear, the response of the sygtern to an initial disturvance

$$
\psi\left(x, t_{0}\right)=e^{i \underline{s} \cdot x},
$$

is

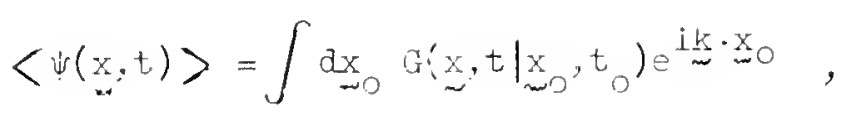

and, since for a homogeneous velocity field, $G\left(\underset{w}{x},\left.t\right|_{0}, t_{0}\right)$ depends on $\underline{x}$ and $\underline{x}_{0}$ in the combination $x_{-}^{-} x_{0}$ only, equation (A.2) can be rewritten

$$
\langle\psi(\underline{x}, t)\rangle=(2 \pi)^{3} \underset{G}{\widetilde{G}}\left(\underline{\underline{x}}, t \mid t_{0}\right) e^{i \underline{i} \cdot \underline{x}},
$$

where

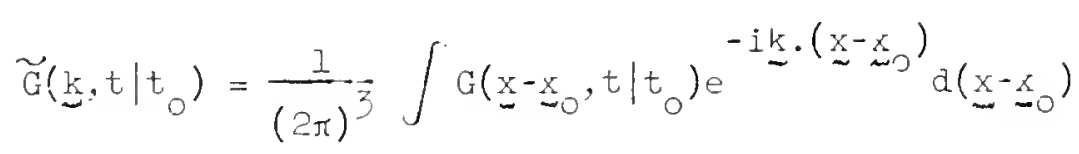

is the Fourier transform of the Green's function $G\left(x_{-},\left.t\right|_{-}, t_{0}\right)$. Equation (A.3) proves the average response matrix of the Fourier modes is diagonal (when the velocity field is hompeneous) and that $(2 \pi)^{3} \tilde{G}\left(\underline{k}, t / t_{0}\right)$ is the averare responise function for note $\mathrm{k}$.

Having established this correspondence, we will now derive an approximate equation for the response funetion by a method parallel to that 
employei by Kraichna $(19 \%)^{*}$ fur the velocity field response function. For aimplicity, we will sifpose heneeforth that the velocity field is also statistically stationary. The modification necessary if the field is not stationary are easily included.

It is convenient to introduce the artifice of cyclic boundary conditions over a large cube of side $L$ in order to expand $\psi(x, t)$ and $\underline{w}_{w}(x, t)$ in Fourier suns rather than Fourier integrals:

$$
\begin{aligned}
& \psi(\underset{\sim}{x}, t)=\sum_{k} \tilde{\psi}(\underset{\sim}{k}, t) e^{i \underline{w} \cdot x} \cdot\left[\tilde{\Psi}(\underset{\sim}{k})=\widetilde{\psi}^{*}(-\underline{k})\right],
\end{aligned}
$$

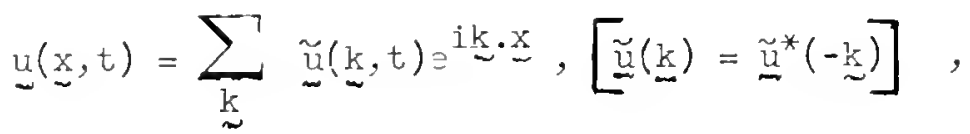

(ci. K., eq. 2.I). Equation (2.2) may be writter

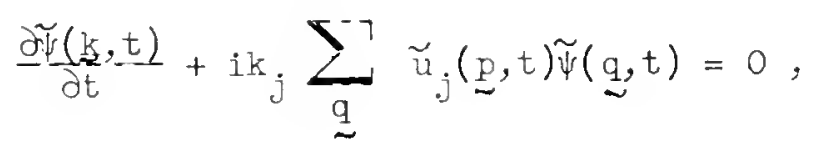

where $p=k-q$. The response function $\tilde{g}(\underline{k}, t)$ for mode $k$ is the solution of $(A .7)$ under the initial conditions

$$
\begin{aligned}
& \widetilde{\Psi}(\underline{\underline{k}}, t) \equiv \tilde{g}(\underset{\sim}{k}, t)=1 \\
& \left.\tilde{\psi}(\underline{q}, t) \equiv \widetilde{\psi}_{\underset{K}{K}}(q, t)=0, \underline{q} \neq \underset{\sim}{k}\right\}^{t}=0 \text {, }
\end{aligned}
$$

\footnotetext{
* This paper will be designated by ' $K$ ' in these Appendices. Some differences in notation shoild be noted: In $\mathrm{K} ., \mathrm{g}(\mathrm{k}, \mathrm{t})$ refers to the velocity field (impulse) response. function and not to the response function for (A.9) below. Also, in $K ., \mathrm{g}$ reiers to an averaged response while, in this appendix, it does not; the average being denoted by $\langle g\rangle$. Further, the notational distinction between a quantity and its Fourier transform is different from that adopted in this paper.
} 
(cf.K. Sec.2.1). By the equation of motion $\widetilde{\psi}_{k}(q, t)$ and the direct interaction approximation, we find (cf. K. eq. 2.24) that for solution (A.8)

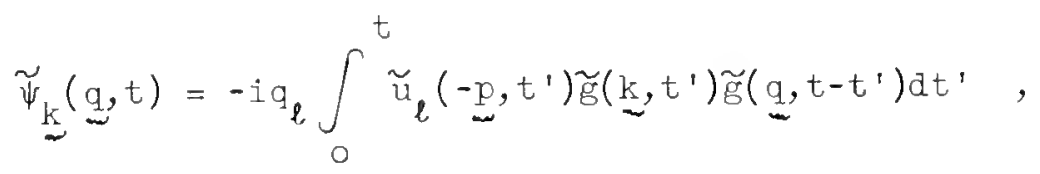

Tnixs by (2.30) we nave

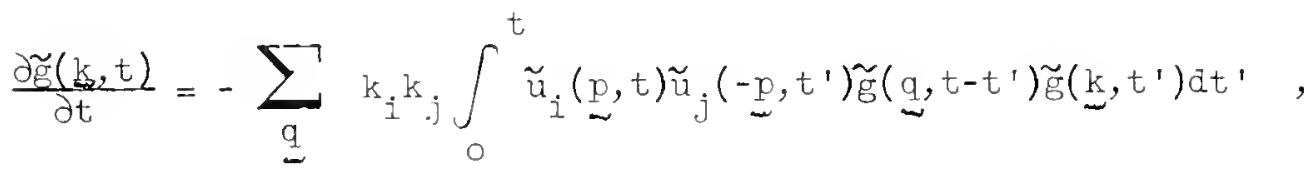

and, on averaging, using the principle of wear statistical dependence (cf. K. Sec. 2.2 and eq. (2.25)), we find

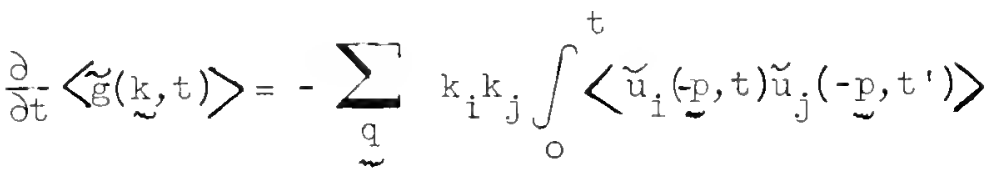

$$
\begin{aligned}
& \left\langle\tilde{g}(\underbrace{q}_{w}, t-t^{\prime})\right\rangle\left\langle\tilde{g}\left(\underline{k}, t^{\prime}\right)\right\rangle d t^{\prime} .
\end{aligned}
$$

Now let us take the limit. Make the transition $L \rightarrow \infty$. Let

$$
\widetilde{U}_{i, j}\left(\underset{m}{k}, t-t^{\prime}\right)=\operatorname{Limit}_{L \rightarrow \infty}\left(\frac{L}{2 \pi}\right)\left\langle\tilde{u}_{i}(\underset{\sim}{k}, t) \tilde{u}_{j}\left(-k, t^{\prime}\right)\right\rangle
$$

so that

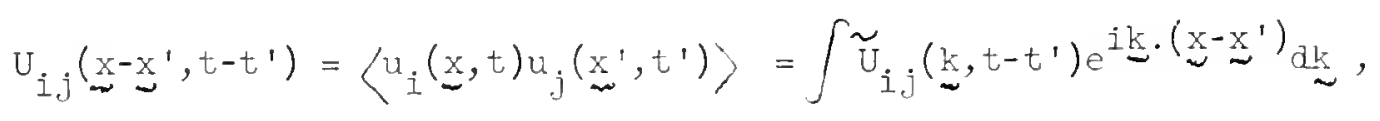

and let

$$
\widetilde{G}(\underset{w}{k}, t)=\underset{L \longrightarrow \infty}{\operatorname{Limit}}\left(\frac{L}{2 \pi}\right)^{3}\langle\tilde{g}(\underset{w}{k}, t)\rangle
$$

so that equations $(A .4,5,8)$ are consistent (cf. eqs. 3.2, 3.3). Then

$$
\frac{\partial \widetilde{G}(k, t)}{\partial t}=-(2 \pi)^{3} k_{i} k_{j} \int_{0}^{t} d t^{\prime} \int d \underline{\sim} \tilde{U}_{i j}\left(\underset{m}{p}, t^{\prime}\right) \tilde{G}\left(\underline{q}, t^{\prime}\right) \widetilde{G}\left(\underset{w}{k}, t-t^{\prime}\right) .
$$


This result can be returned to physical space by writing it as

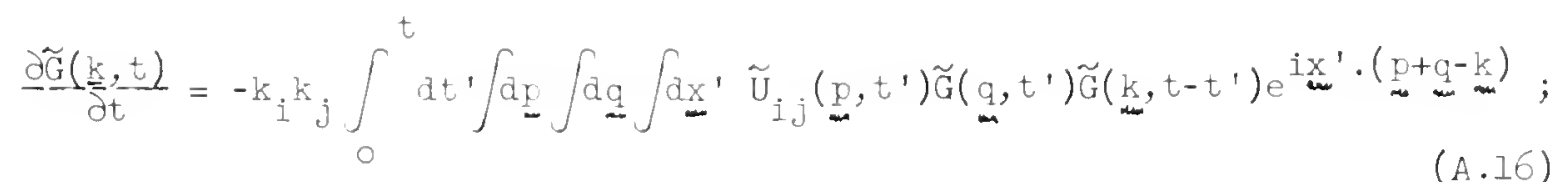

i.e. (cf. A.4, 13)

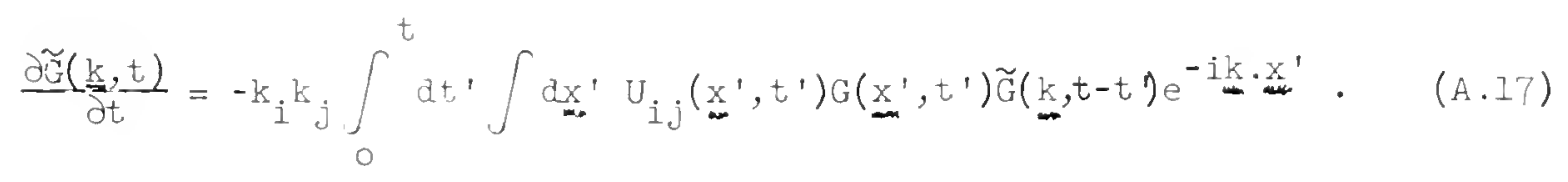

Using (A.4) again, this can be rewritten

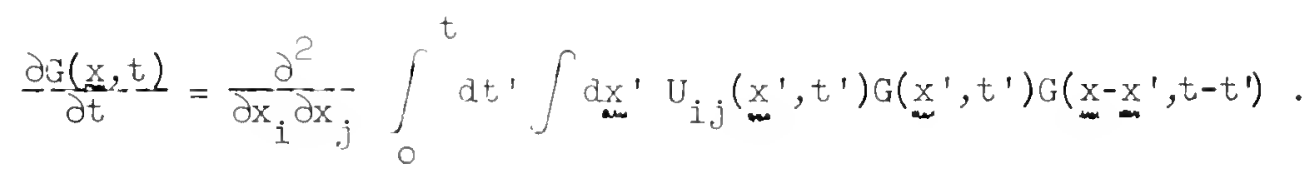

We no's give an alternative derivation of (A.15) based on sumning selected terins from all onders of the fomal expansion. For simplicity we will treat the one dimensional case only. Then (2.2) is replacel by

$$
\frac{\partial \psi(x, t)}{\partial t}=-\frac{\partial}{\partial x}[u(x, t) \psi(x, t)] .
$$

On maing expansions analagous to (A.5, 6), we obtain from (A.19)

$$
\frac{\partial \tilde{\psi}(k, t)}{\partial t}=-i k \sum_{q} \tilde{u}(p, t) \tilde{\psi}(q, t)
$$

where $p=k-q$. (We will again suppose the mean flow $\tilde{u}(0, t)$ is zero.) The fommal solution for the response function for mode $k$ (cf. A.8) is 


$$
\begin{aligned}
\tilde{g}(k, t)=1 & -\sum_{\ell+m=0} k(k-\ell)[1 * \tilde{u}(\ell) * \tilde{u}(m)]_{t} \\
& +1 \sum_{\ell+m+n=0}^{-1} k(k-\ell)(k-\ell-\tilde{n})[1 * \tilde{u}(\ell) * \tilde{u}(m) * \tilde{u}(n)]_{t} \\
& +\sum_{\ell+m+n+r=0} k(k-\ell)(k-\ell-m)(k-\ell-m-i)[1 * \tilde{u}(\ell) * \tilde{u}(m) * \tilde{u}(n) * \tilde{u}(r)]_{t} . .,
\end{aligned}
$$

where the * denotes the process of convolution: -

$$
\left[f^{*} g\right]_{t}=\int_{0}^{t} f\left(t^{\prime}\right) g\left(t-t^{\prime}\right) d t^{\prime}
$$

To determine the response $\widetilde{\psi}_{k}(q, t)$ indxced in moje q by the initial excitation in mode $\mathrm{k}$. we can write (A.20) in the form

$$
\tilde{\psi}_{k}(q, t)+i q \int_{0}^{t} d t^{\prime} \tilde{u}\left(-p, t^{\prime}\right) \tilde{g}\left(k, t^{\prime}\right)=-i q \sum_{l \neq-p}^{-\urcorner} \int_{0}^{t} \tilde{u}\left(l, t^{\prime}\right) \widetilde{\psi}_{k}\left(q-l, t^{\prime}\right) d t^{\prime},
$$

and iterate starting with a zero th approximation derived by setting $\tilde{\psi}_{\mathrm{k}}=0$ on the right, followed by a first approximation obtained by substituting the zero $^{\text {th }}$ approsimation for $\tilde{\psi}_{k}$ into the right-hand side of (A.2?), and so on. In this way, we find

$$
\begin{aligned}
\tilde{u}(p, t) \tilde{\Psi}_{k}(q, t)= & -i q u(p, t)[I *\{\tilde{u}(-p) \tilde{g}(k)\}]_{t} \\
& -q u(p, t) \sum_{\ell}^{\urcorner}(q-\ell)[I * \tilde{u}(\ell) *\{\tilde{u}(-p-i) \tilde{g}(k)\}]_{t} \quad(A \cdot 23) \\
& +i q u(p, t) \sum_{\ell, m}(q-\ell)(q-\ell-m)[I * \tilde{u}(\ell) * \tilde{u}(m) *\{\tilde{u}(-p-\ell-m) \tilde{g}(k)\}]_{t}
\end{aligned}
$$


where the sums exclude indices for which $\ell, \ell+m, \ell+m+n, \ldots \ldots . \ldots$ are equal to $-p$.

So far, the analysis is exact. Now we make a selection of terms. Equation (A.7) shows that to each interaction which, through the agency of $\tilde{u}(p)$, induces $a$ rate of change in $\widetilde{\Psi}(k)$ proportional to $\widetilde{\Psi}(k-p)$, there is a conjugate interdetion which, through the agency of $\tilde{u}(-p)$, induces a rate of change in $\tilde{\psi}(k-p)$ which is proportional to $\widetilde{\psi}(k)$. There are, of course, less direct couplings between $\widetilde{\psi}(k)$ and $\widetilde{\psi}(k-p)$ wich involve more than one intermediate $\tilde{u}$ mode, but, in the fomnal solution (A.2l) for $\tilde{g}(k, t)$ and the expression $(A .23)$ for $\tilde{u}(p, t) \Psi_{K}(q, t)$, we will only retain a term if all the interactions of which it is composed orcur in conjugate pairs. (We note that this automatically excludes all terms with an adi number o: u factors.)

We can also exolain our selection rule by a diagramatic representation of the terns composing the series (A.21). A typical terin containing $2 n$ u-fictors will be said to be of order $n$. The wave numbers of the $\widetilde{\psi}(k-p)$ modes involved in the interaction with $\tilde{\psi}(\mathrm{k})$ forin the segments of the oase line of the diagram (see figure 1 ) and the ingoing arrow on the left marked $k$ represents the mode $\widetilde{\psi}(k)$ itself. Tho noxt segment, marked $(k-\ell)$, represents the $\tilde{\psi}(k-\ell)$ mode with which it interacts, the $\tilde{u}(\ell)$ mode concernea in the interaction being represented by the loop marked $\&$ leaving the base line. The following intersections of loop and line have a similar meaning. The selection mule ensures that to every vertex A involving $\tilde{\psi}(s), \tilde{u}(\ell), \tilde{\psi}(s-\ell)$ there is a conjugate vertex $A^{\prime}$ involving $\tilde{\psi}(s-\ell), \widetilde{u}(-\ell)$, $\tilde{\psi}(s)$. Thus the sum of all the $\tilde{u}$-factors inrolved at vertices between $\mathrm{A}$ and $A^{\prime}$ is zero; i.e. there is an even number of such vertices and these 
50.

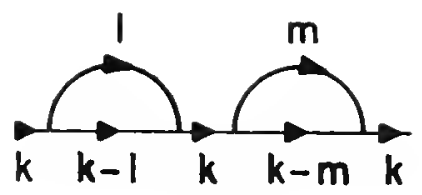

(a)

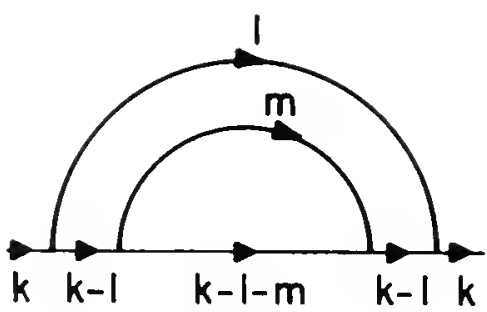

(b)

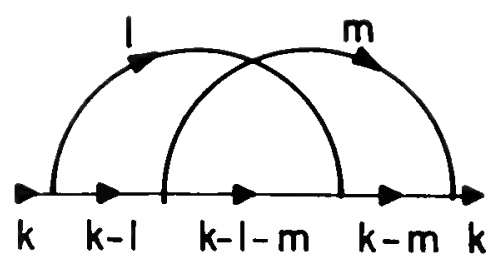

(c)

F1g. 1: Diagrams for $\mathrm{n}=2$ representing three nonzero terms of $\left[I^{*} u(\ell) * u(m) * u(n) * u(r)\right]_{t}$ of equation $(A \cdot 21)$. (a) $\ell+m=n+r=0$, (b) $l+r=m+n=0$, (c) $l+n=m+r=0$. 
are conjugate in pairs. Thus the lines joining conjugate vertices do not cut each other.

Two consequences of the selection rule should be noted. First, the only. terms of the Pormal expanion which are incladel are those wich involve pairs $\tilde{u}(\ell), \tilde{u}(-\ell)$. These terms are the same in normal and non-normal distributions having the same covariance. Secondly, not all ternis inrolving pairs $\tilde{u}(\ell), \tilde{u}(-\ell)$ are inoluked; for they will not be con.jugate verices if the loop joining them cuts the loop joining another pair. For example in figure $I$ all the interactions are shown for $\mathrm{n}=$ ? and, by the selection rule, we retain only $I(a)$ and $l(b)$. Consider now a general terr in the series (A.23). $i q(q-\ell)(q-\ell-m) \ldots \tilde{u}(p, t)[\underline{I} * \tilde{u}(\ell) * \tilde{u}(m) * \tilde{u}(n) * \ldots *\{\tilde{u}(-\tilde{p}-\ell-m-n \cdots \ldots) \tilde{g}(k)\}]_{t}$. The initial $\tilde{u}(p, t)$ term must find a mate $\tilde{u}(-p)$ within the convolution produet. Suppose it is not $\tilde{u}(-p-\ell-m \ldots \ldots \ldots)$. It cannot be any of the even placed terns $\tilde{u}(m) . . . . . . .$. , for, if it were, there would be an odd number of vertices on the diagram between $\tilde{u}(p)$ and $\tilde{u}(-p)$ and these could not all find mates without cutting the loop joining $\tilde{u}(p)$ and $\tilde{u}(-p)$. The nate for $\tilde{u}(p)$ must therefore be one of the ord placed terms $\tilde{u}(\ell), \tilde{u}(n), \ldots$ There is then an even number of vertices on the diagram between $u(p)$ and $u(-p)$ and these vertices can and, by the selection mule, must be joinea withoit cutting the loop joining $\tilde{u}(p)$ and $\tilde{u}(-p)$; i.e. the sun of the wave numbers $\ell+m+\ldots \ldots$. for vertices lying between $\tilde{u}(p)$ and $\tilde{u}(-p)$ musi be zero; in other words, the sum of these intermediate wave numbers together with the wave number -p for the mate of $p$ itself must equal $-p$. But these 
terms are precisely the ones excluted in the sunmations of (A.26). Hence we have reached a contradiction. Thus, the mate for $\tilde{u}(p)$ must be $\tilde{u}(-p-\ell-m .$.$) and the sum \ell+m+\ldots \ldots$ of all internediate wave-numbers mixt be zero. Now compare the general term of the series (A.23) for $\tilde{u}(p, t) \widetilde{\psi}_{k}(q, t)$ with the corresponding term [as derived from (A.2l)] for the series for

$$
-i q \tilde{u}(p, t)[\{\tilde{u}(-p) \tilde{g}(k)\} * \tilde{g}(q)]_{t}
$$

It will be readily seen from the above argunent that, under the selection rule, the two series are identical; i.e.

$$
\tilde{u}(p, t) \tilde{\psi}_{k}(q, t)=-i q \int_{0}^{t} \tilde{u}(p, t) \tilde{u}\left(-p, t^{\prime}\right) \tilde{g}\left(k, t^{\prime}\right) \tilde{g}\left(q, t-t^{\prime}\right) d t^{\prime} .
$$

Hence, by (A.20), and the principle of weak statistical dependence (K. Sec. 2.2),

$$
\frac{\partial\langle\tilde{g}(k, t)\rangle}{\partial t}=-k \sum_{q} q \int_{0}^{t} d t^{\prime}\left\langle\tilde{u}(p, t) \tilde{u}\left(-p, t^{\prime}\right)\right\rangle\left\langle\tilde{g}\left(k, t^{\prime}\right)\right\rangle\left\langle\tilde{g}\left(q, t-t^{\prime}\right)\right\rangle, \quad(A \cdot 24)
$$

as berore. (Tnis proop generalizes easily to 3 -dimensions.)

As further confirmation, we can show, by the direct counting of diagrams, that for large $k\left(\gg \ell_{0}^{-1}\right)$ the selection criterion gives

$$
\langle\tilde{g}(k, t)\rangle=J_{I}\left(2 k v_{1} t\right) / k v_{1} t
$$

which (cf. eq. 2.37) is also the solution to (A.24) under these circumstances. For large $k$, all the terns $k-\ell, k-\ell-m$, etc. of (A.2l) are equal to $k$ with negligible error. Also, any convolution term of order $n$ is asynptotically equal to 


$$
\left(v_{1}+\right)^{2 n} /(-n):
$$

(since, as we can show a posteriori, $\langle\tilde{E}(k, t)\rangle$ is negligibly small unless $\left.t<\ell / v_{0}\right)$. Hence

$$
\left.\langle\tilde{g}(k, t)\rangle=\sum_{0}^{\infty}(-1)^{n} N_{n} \cdot k v_{1} t\right)^{2 n} /(2 n) !
$$

where $\mathbb{N}_{n}$ is the number of non-intersecting diagrains of order $n$.

To deternine $N_{n}$, we introduce the idea of a block. A diagram will be said to be composed of $s$ blocks if there exist s particular conjurgte pairs $A_{1} A_{1}{ }^{\prime}, A_{2} A_{2}{ }^{\prime} \ldots . . . A_{S} A_{S}{ }^{\prime}$ with the following properiy: that both vertices of every other conjugate pair B B' lie between $A_{r} A_{n}$ ' for some choice of $r$. In other words, there are no loops in the diagram wlich (topologlcally) lie above any of the pairs $\mathrm{A}_{r} \mathrm{~A}_{r}{ }^{\prime}$. For examples, see figure 2, in which the 5 loops composing the outline of the blocks are identified by heavy lines. Suppose the number of diagrams of order $n$ composed of $\mathrm{s}$ blocks is $N_{n, s}(I \leq s \leq n)$. We proceed by inkuction. We consider an $s$ bloci diagram of order $n-1$, and add an additional loop whose lett-hend vertex $L$ lies to the left of the entire s blook diagram. There are (s+l) possible positions for the right-hand vertex $R: P_{1}, P_{2} \ldots \ldots \ldots . . . P_{s+1}$ (see figlre 3), and, if we choose $\mathrm{P}_{r}$, the diagram of order 11 thereby generated has $r$ blocks. Remembering that $L$ is always to the left of the entire diagram of order $(n-I)$, it is not difficult to see that every diagram of omder $n$ can be generated without duplication by applying this process to every $(n-1)$ order diagram. Since an $(n-1)$ order s block diagram produces one diagran each of $p$ blocks $(p=1,2 \ldots \ldots . ., s+1)$, we see that 
54.
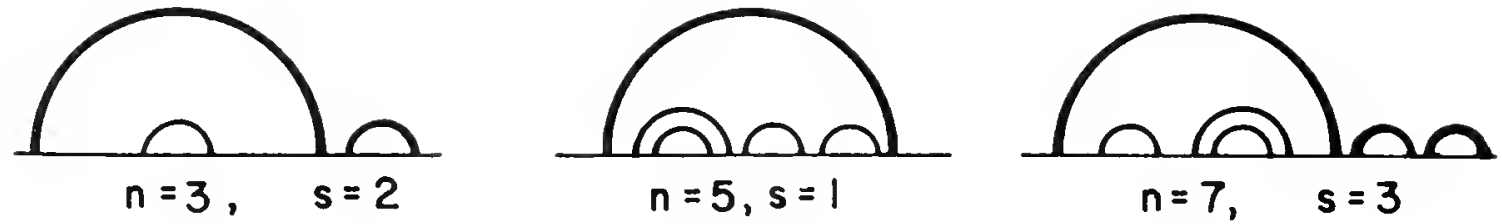

Fig. 2: Illustrations of block structure of diagrams. Outlines of blocks are identified by heavy lines.

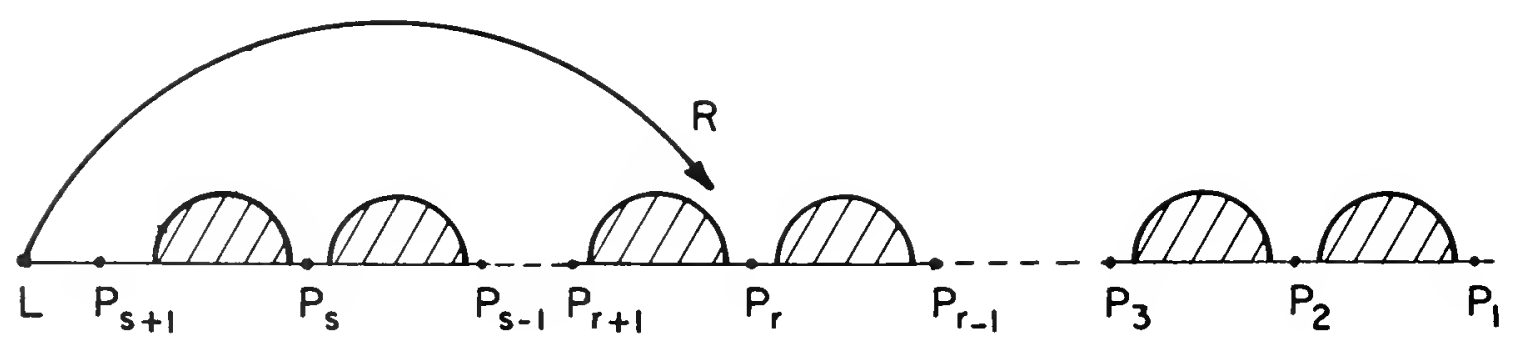

Fig. 3: The generation of a diagram of order n from a

diagram of order $n-1$. Shaded areas denote blocks. 


$$
N_{n, S}=\sum_{r=S-1}^{n-1} N_{n-1, r}
$$

with the convention $\mathrm{N}_{\mathrm{n}, \mathrm{O}}=0$. It follows that

$$
N_{n, r}=\frac{r(2 n \cdots r-1) !}{n !(n-r) !}
$$

and, since every $n$ order diagram gives rise to one, and only one, $n+l$ order, 1 block diagran, we nave

$$
N_{n}=N_{n+1,1}=\frac{(2 n) !}{n !(n+1) !}
$$

Therefore, by (A.25),

$$
\langle\tilde{g}(k, t)\rangle=\sum_{0}^{\infty}(-1)^{n} \frac{\left(k v_{1} t\right)^{2 n}}{n !(n+1) !}=\frac{J_{1}\left(2 k v_{1} t\right)}{k v_{1} t} .
$$




\section{APPENDIX B:}

\section{Derivation of equation (3.18)}

As in appendix $A$, we will suppose that the velosity field is homogeneous and stationary, and consider homogeneous forms of $\psi_{1}$ and $\psi_{2}$. We again adopt cyclic boundary conditions and expansions of the form $(A .5,6)$;

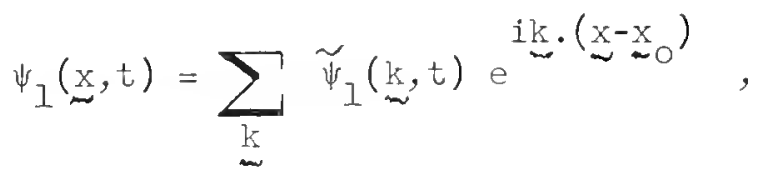

$$
\begin{aligned}
& \psi_{2}(\mathrm{y}, \mathrm{s})=\sum_{m} \widetilde{\psi}_{2}\left(\mathrm{k}_{\sim}, \mathrm{s}\right) \mathrm{e}^{i \mathrm{k} \cdot\left(\mathrm{y}-\mathrm{y}_{0}\right)}, \\
& u(x, t)=\sum_{w} \tilde{w} \underset{w}{\tilde{u}}(\underset{w}{k}, t) e^{i k \cdot x}
\end{aligned}
$$

(cf. K. eq. 2.I and footnote to Appendix A). Then equation (2.2) for the $\psi_{1}$ field may be written

$$
\frac{\partial \widetilde{\Psi}_{I}(\underline{k}, t)}{\partial t}+i k_{i} \sum_{\underline{q}} \widetilde{u}_{i}(\underline{p}, t) e^{-i \underline{p} \cdot x_{0}} \widetilde{\psi}_{I}(\underline{q}, t)=0
$$

and, for the $\psi_{2}$ field,

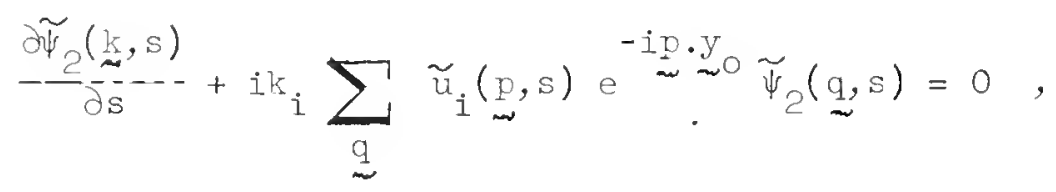

where $p=k-q$. By $(B .4)$ we have (cf. K. eqs. 3.4, 3.5)

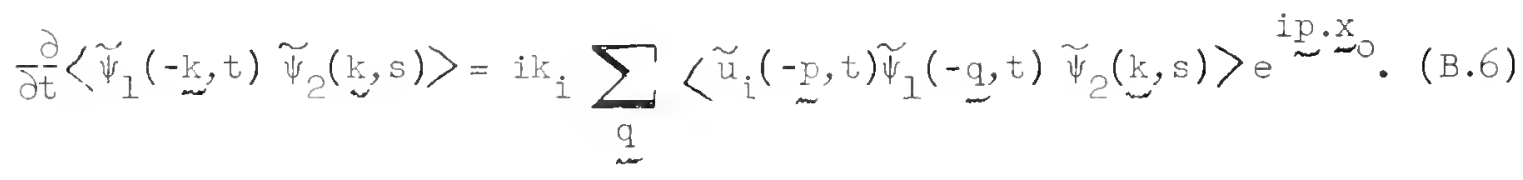


Now, in the limit $\mathrm{L} \rightarrow \infty$, the direct interaction approximation (K. Sec. 2.4) gives

$$
\begin{aligned}
& \left\langle\tilde{u}_{i}(-\underline{p}, t) \widetilde{\psi}_{1}(-\underline{q}, t) \widetilde{\psi}_{2}(\underline{k}, s)\right\rangle
\end{aligned}
$$

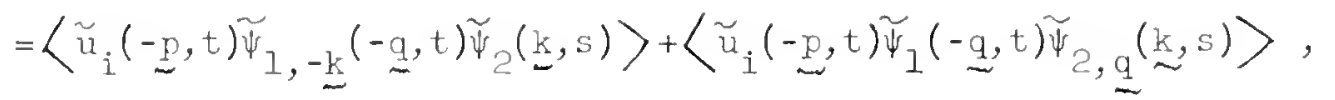

where (cf.eq. A.9 and K. Sec. 3)

$$
\begin{aligned}
& \tilde{\psi}_{1,-\underline{\sim}}(\underline{-q}, t)=\int_{t_{0}}^{t} i q_{j} \tilde{u}_{j}\left(\underline{p}, t^{\prime}\right) \widetilde{\psi}_{1}\left(-\underset{\sim}{k}, t^{\prime}\right) \tilde{g}\left(\underset{\sim}{q}, t-t^{\prime}\right) e^{-i \underset{\sim}{p} \cdot \sim_{0}} d t^{\prime},
\end{aligned}
$$

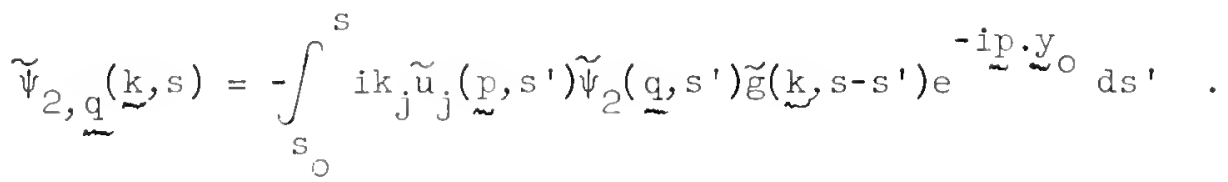

Thus, applying the weak statistical dependence principle (K. Sec. 2.?), we find that, in the limit $L \rightarrow \infty$, (ct. eqr. A.12, 13, 14)

$$
\begin{aligned}
& \frac{\partial}{\partial t}-\widetilde{R}\left(k, t,\left.s\right|_{w 0} ^{r}, \tau_{0}\right)= \\
& -(2 \pi)^{3} k_{i} k_{j} \int_{0}^{t} d t^{\prime} \int d \underline{\sim} \widetilde{U}_{i j}\left(\underset{\sim}{p}, t^{\prime}\right) \widetilde{G}\left(\underset{\sim}{q}, t^{\prime}\right) \widetilde{R}\left(\underset{\sim}{k}, t-t^{\prime}, s \mid{\underset{\sim}{0}}_{0}, \tau_{0}\right)
\end{aligned}
$$

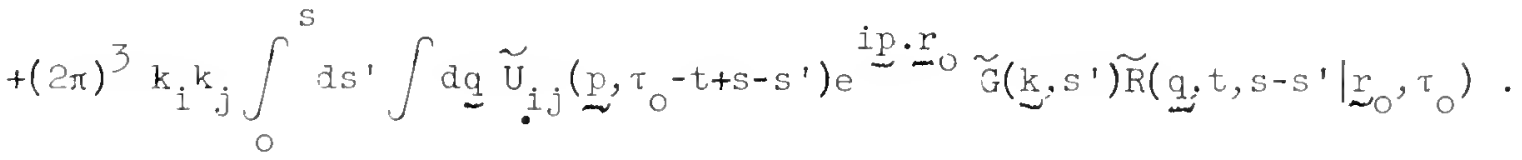

Returning to $x$-space by the argunents of (A.15) to (A.I8) this becomes 
$\frac{\partial}{\partial t} R\left(r, t, s \mid r_{0}, \tau_{0}\right)=$

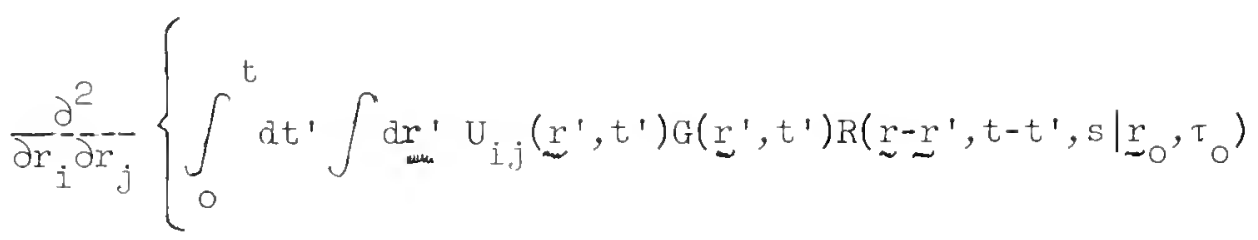

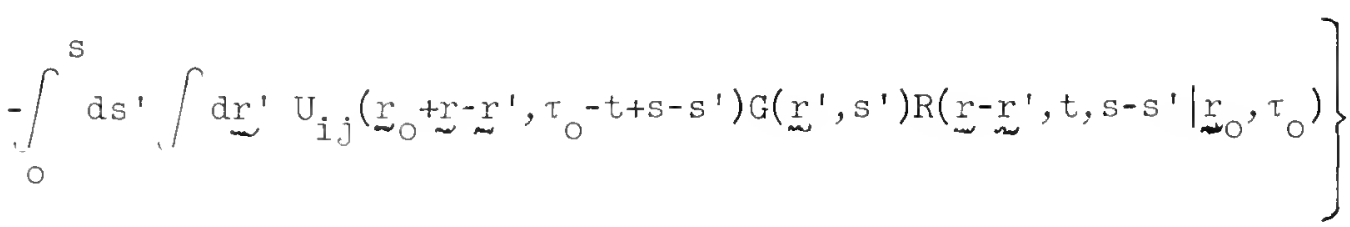

which is equivalent to (3.18).

An alternative derivation of (B.II) can be given which is based on summing selected temns from all orders of the formal expansion of $R$. We will not discuss this method in detail, but we will describe how it differs from the similar method of appendix $A$, and for this purpose we will once more discuss the one dimensional case. The formal solution of (B.4) under the initial contition $\psi_{1}(x, t)=\delta\left(x-x_{0}\right)$ is given by

$$
\begin{aligned}
& L^{3} \widetilde{\Psi}_{I}(-k, t)=I+i \sum_{\ell} k[I * \widetilde{u}(\ell)]_{t} e^{-i \ell x_{0}}-\sum_{\ell, m} k(k+\ell)[I * \tilde{u}(\ell) * \tilde{u}(m)]_{t} e^{-i(\ell+m) x_{0}} \\
& -i \sum_{\ell, m, n} k(k+\ell)(k+\ell+m)[1 * \tilde{u}(\ell) * \widetilde{u}(m) * \tilde{u}(n)]_{t} e^{-i(\ell+m+n) x_{0}}+\ldots \ldots
\end{aligned}
$$

Multiply this series term by term by the similar expansion for $L^{3} \widetilde{\psi}_{2}(k, t)$ and average, retaining only conjugate interactions. Now, however, the mate of $\tilde{u}(p)$ in one series may occur in a term of the other series, and it is precisely such interactions which, on the direct interaction approxi- 
mation. Give rise to the correlation between $\psi_{1}$ ani $\psi_{2}$. As in Arrenix A, a diagramatic representation may be made of the formal expansions. Now, however, there are two base lines, one for $\Psi_{1}$ and one for $\tilde{\psi}_{2}$. The $\tilde{u}$ modes are represented by loops joining conjugate vertices either on the same or on different base lines, and, as in Appendix A, these loops cannot cross. A typical term of the series for $\tilde{\Psi}_{1}(-\mathrm{k}) \tilde{\psi}_{2}(\mathrm{k})$ is shown aiagramatically in figure 4: it is obtained from the product of a second order term in $\widetilde{\Psi}_{1}(-k)$ and a Pourth order term in $\widetilde{\psi}_{2}(k)$.

On the basis of this selection rule of Appendix A, we will now recover the solution of Sec. 3.3 for large k. Under these circumstances, all the terms $k+\boldsymbol{l}, k+\boldsymbol{l}+\mathrm{m}$, etc. of (B.I2) are equal to $k$ with negligible error. Also, a pair of conjugate vertices will, asymptotically, give a contribution of

$$
\sum_{\ell} \tilde{u}(-\imath, 0) \tilde{u}(\imath, 0)=v_{1}^{2},
$$

if both vertices refer to $\widetilde{\psi}_{1}$ or $\widetilde{\psi}_{2}$, and give a contribution of

$$
\sum_{\ell} \tilde{u}\left(-\ell, t_{0}\right) e^{1 \ell x_{0}} \tilde{u}\left(\ell, s_{0}\right) e^{-i \ell y_{0}}=\left\langle u\left(x_{0}, t_{0}\right) u\left(y_{0}, s_{0}\right)\right\rangle \equiv U_{1 I}\left(r_{0}, \tau_{0}\right),
$$

if one vertex refers to $\tilde{\psi}_{1}$ and the other to $\tilde{\psi}_{2}$. Hence

$$
\begin{aligned}
& \left\langle\tilde{\Psi}_{1}(w, t) \tilde{\Psi}_{2}(\mathrm{k}, \mathrm{s})\right\rangle= \\
& \sum_{n, m, \ell} \frac{(-1)^{m+n}}{(\ell+2 m) !(\ell+2 n) !}\left[\frac{U_{11}\left(r_{0} \tau_{0}\right)}{2}\right]^{\ell} N_{i m ; n ; \ell}\left(k v_{1} t\right)^{\ell+2 m}\left(k v_{1} s\right)^{\ell+2 n},
\end{aligned}
$$


60.

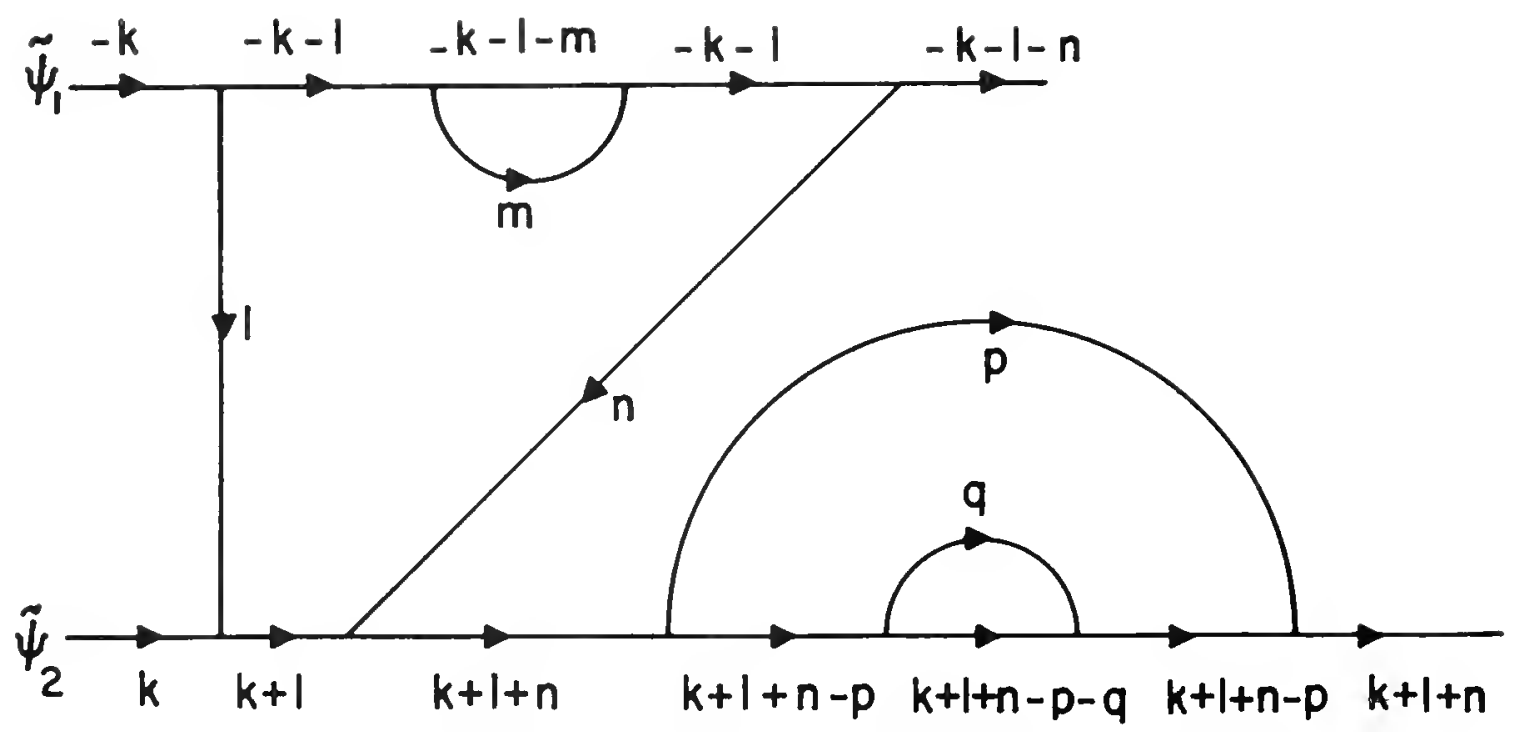

Fig. 4: A typical term in the formal expansion of $\left\langle\widetilde{\psi}_{1} \widetilde{\psi}_{2}\right\rangle$.

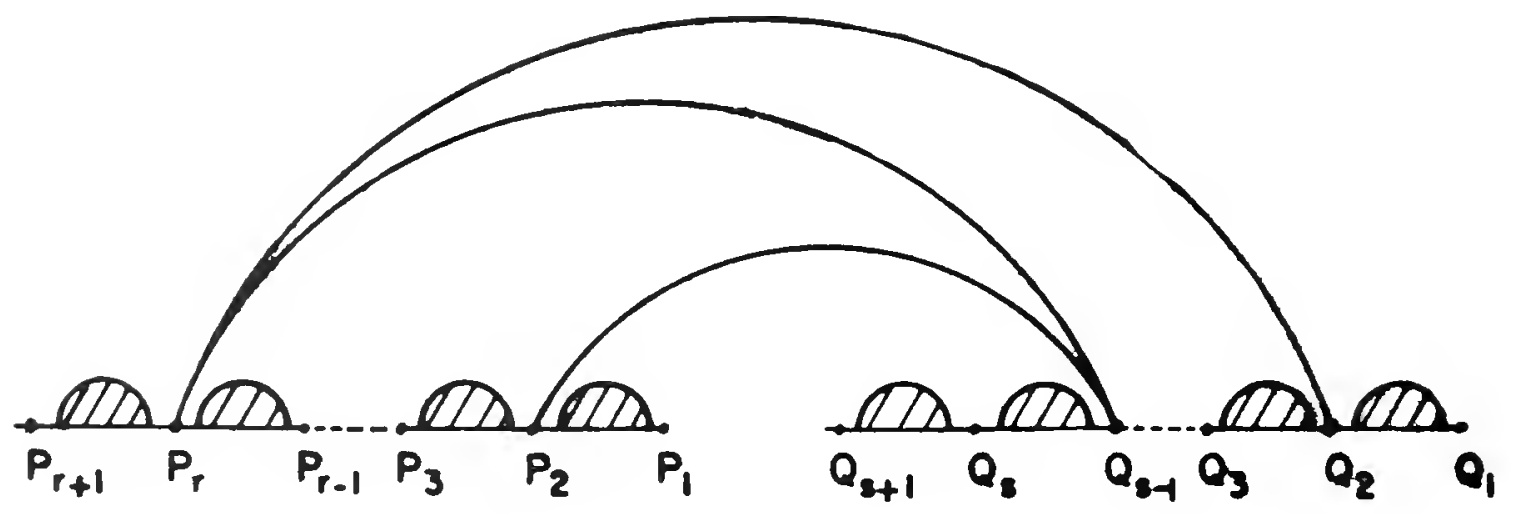

Fig. 5: A general term $\left\langle\tilde{\psi}_{1} \tilde{\psi}_{2}\right\rangle$ showing location of termini of links. Blocks are shaded. 
where $\mathbb{N}_{m ; n} \mathfrak{l}$ is the number of non-intersecting liagrains with $\ell+2 m$ points on the $\tilde{\Psi}_{1}$ base Iine, $\ell+2 n$ points on the $\tilde{\psi}_{2}$ base line and $\ell$ linirs between them.

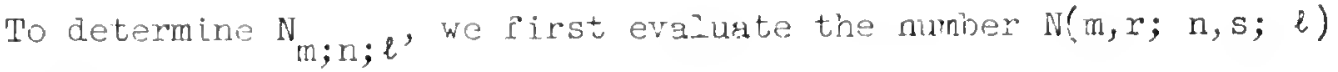
of diagrans in whish the $\ell+$ mon points lying on the $\widetilde{\psi}_{I}$ base line are composed of $r$ blocks and $\ell$ links, and the $\ell+2 n$ points on the $\tilde{\psi}_{2}$ base Iine are composed of s blocks an $\ell$ linis. For example, figure 4 shows one diagran counted in $N(2,1 ; 4,1 ; 2)$. There are $(r+1)$ possib?e locations $\mathrm{P}_{1}, \mathrm{P}_{2}, \ldots \ldots \ldots \mathrm{P}_{\mathrm{r}+1}$ for the termini of the $\ell$ links on the $\tilde{\Psi}_{1}$ base Iive and $(s+1)$ possible locations $Q_{1} ; Q_{2}, \ldots \ldots \ldots Q_{S+1}$ for the termini on the $\widetilde{\psi}_{2}$ base line (see figure 5), and any n!umber of termini can be placed at any of these locatinn. There are therefore $(r+\ell) ! / r ! \ell$ : way: of placing the $\ell$ ternini on the $\Psi_{1}$ base line and $(s+\ell) ! / s ! \ell !$ ways of placing the other $l$ termini on the $\tilde{\psi}_{2}$ base line. There is only one way of joining the $\ell$ ternini on each base line with non-intersecting liniss. Hence, in all, there are

$$
\frac{(r+\ell) !}{r ! \ell !} \frac{(s+\ell) !}{s ! \ell !}
$$

ways of linking the base lines. As we have seen (cf. A.23), there are

$$
\frac{r(2 m-r-1)}{m !(m-r) !} ! \quad \frac{s(2 n-s-1) !}{n !(n-s) !}
$$

ways of forning the blocks on base lines. Hence.

$$
N(m, r ; n, s ; \ell)=M(m, r ; \ell) M(n, s ; \ell) \text {, }
$$


62.

$$
M(m ; r ; \ell)=\frac{(r+\ell) !}{r ! \ell !} \frac{r(2 r n-r-1) !}{m !(m-r) !} .
$$

It forlo's that

$$
N_{m ; n ; \ell}=\left[\sum_{r=1}^{m} M(m, r ; \ell)\right]\left[\sum_{s=1}^{n} M(n, s ; \ell)\right] .
$$

Now, let

$$
S_{m, l}=\ell ! m ! \sum_{r=1}^{m} M(m, r ; l)=\sum_{0}^{m-1} \frac{(m+l-r) !}{r !} \frac{(m+r \cdot 1) !}{(m-r-1) !} .
$$

From the calculations of Appendix A (cf. A.23, A.29), we know that

$$
S_{m,-1}=(2 n-I) ! / m !, S_{m, 0}=(2 m) ! /(m+1) !,
$$

and it is easy to establish from (B.I6) that

$$
S_{i n, \ell}+S_{m+1, \ell-1}=(2 m+\ell) S_{m, \ell-1}+\ell S_{m+1, \ell-2} \text {. }
$$

By this recurrence relation; we may prove from (B.17) by induction that,

$$
S_{m, \ell}=\frac{(\ell+1) !(\ell+2 i n) !}{(m+\ell+1) !}
$$

i.e.

$$
\left.\sum_{r=1}^{m}\right\urcorner(m, r ; \ell)=\frac{(\ell+1)(\ell+2 m) !}{m !(m+l+1) !} .
$$


Returning to (B.13) and (B.15) we find

$$
\begin{aligned}
& \tilde{\psi}_{I}(-k, t) \widetilde{\psi}_{2}(k, s) \\
& =\sum_{n, m, \ell} \frac{(-1)^{m+n}(\ell+1)^{2}}{m ! n !(m+\ell+1) !(n+l+1) !}\left[\frac{U_{11}\left(r_{0}, \tau_{0}\right)}{v_{1}^{2}}\right]^{\ell}\left(k v_{1} t\right)^{\ell+2 m}\left(k v_{1} s\right)^{\ell+2 n} \\
& =\sum_{l}(\ell+1)^{2}\left[\frac{U_{11}\left(r_{0}, \tau_{0}\right)}{v_{1}^{2}}\right]^{\ell} \frac{J_{\ell+1}\left(2 k v_{1} t\right)}{k v_{1} t} \frac{J_{\ell+1}\left(2 k v_{1} s\right)}{k v_{1} s},
\end{aligned}
$$

which is equivalent to (3.31). 
Batchelor, G.K., 1950.

Batchelor, G.K., 1952 a.

Batchelor, G.K., $1952 b$.

Batchelor, G.K., 1953

Corrsin, S., 1960.

Kolmogorov, A.N., 1941.

Kraichnan, R.H., 1959

Kra1chnan, R.H., 1960.

Reid, W.H., 1955.

Richardson, L.F., 1926.
"The application of the similarity theory of turbulence to atmospheric diffusion". Quart. J. Roy. Met. Soc. 76, 133-146.

"Diffusion in a field of homogeneous turbulence II: The relative motion of particles".

Proc. Camb. Ph1l. Soc. 48, 345-363.

"The effect of homogeneous turbulence on material lines and surfaces".

Proc. Roy. Soc. Lond. A, 213, 349-366.

"The theory of homogeneous turbulence". Cambridge: at the University Press.

"Progress report on some turbulent diffusion research".

Proc. Symposium on Atmospheric Diffusion and Air Pollution, held at Oxford, 1958, p. 161-164. Academic Press.

"The local structure of turbulence in an incompressible viscous fluid for very large Reynolds numbers".

C.R. Acad. Scl. U.R.S.S. 30, 301.

"The structure of isotropic turbulence at very high Reynolds numbers".

J. Fluid Mech. 5, 497-543. This paper is referred to as 'K.' in the appendices.

"Dynamies of non-linear stochastic systems". New York University, Institute of Mathematical Sciences, Division of Electromagnetic Research, Report No. HT-7.

"On the stretching of material lines and surfaces in isotroplc turbulence with zero fourth cumulants".

Proc. Camb. Phil. Soc. 51, 350-362.

"Atmospheric diffusion on a distance-neighbour graph".

Proc. Roy. Soc. Lond. A, 110, 709-737. 


$$
65 .
$$

Roberts, P.H., 1957

Roberts, P.H. and

Urse11, H.D., 1959

Saffman, P.G.

Taylor, G.I., 1921

Watson, G.N., 1944
"On the application of a statistical approximation to the theory of turbulent diffusion". J. Rat. Mech. and Anal. 6, 781-800.

"Random walk on a sphere and on a Riemannian manifold".

Phil. Trans。 Roy. Soc. Lond. A, 252, 317-356.

"An approximate calculation of the Lagrangian correlation coefficient for turbulent diffusion". Unpublished.

"Diffusion by continuous movements". Proc. Lond. Math. Soc., 20, 196.

"A treatise on the theory of Bessel functions". and edition. Cambridge, at the University Press. 


\section{DNITIAL DISIRIBUTION LIST FOR RESEARCH RETORTS}

Contract No. N(onr)285-33, Task No 062-206

(ONE copy unless otherrise noted)

Chief of Naval Research Department of the Navy Washington 25, D.C. Attn: Codes 438

411
461
466

Commanding officer

Offlce of Naval Research Branch Orfice

495 Sumer street

Boston 10, Massachussets

Commanding office

office of liaval Research

Branch office

The John Crerar Iflbrary Bldg.

86 East Randolph Street

Chicago 1, Illinols

Cormanding officer

Office of liaval Research

Branch office

346 Broadway

New York 13, New Yor

Commanding orflcer

Office of Navel Research

Brench Office

1030 East Green Street

Pasadena 1, Califormia

Conmanding offlcer

Offlce of Navel Research

Navy 100 , Fleet Post Orfice

New York, New York

Attn: I. M. M.P. Tulin

Drector

Naval Research Laboratory

Washington 25, D.C.

Attn: Code 2000 (Tech. Info. off.)

Chief, Bureau of Aeronautics Departinent of the Navy

Washington 25, D.C.

Attn: Codea AD-3 (Aero \& Fydro.Br.) RS (Research Div.) TD-42(Library Sect.)

Chief, Bureau of Ordnance

Department of the Navy

Washington 25, D.C.

Attn: Codes ReO (Director, Ord. Sel.) Ad3 (Tech. Lib. Br.)

Chief, Bureau of Ships

Department of the Navy

Washington 25, D.C.

Attn: Codes 106 (Tech. Asst. to Chief)

310 (Res. \& Dev. D1v.)

312 (Tech. Lib.)

442 (Sci. \& Res.)

370 (Appl. Scl. Div.)

425 (Adv. Conc. Br.)

Cormanding officer and Director

David Taylor Hodel Rasin

Washinston 7, D.C

Attn: Codes 142 (Library Er*)

500 (Tech. Dir. Bydronech. Lab.)

513 (Contract Res, Admin.)

550 (Underwater Acoustica Div.)

Chlef, Burear of Yarts and Docks

Department of the Navy

Washington 25, D.C.

Attn: Code D 400 (Res. Div.)

\section{Commander}

Vaval Ordnance Iaboratory

White Oak, Waryland

Attn: Desk DA (Assoc. Tech. Mr. Aeroball.) HL. (Library Div.)

Cormander

Naval Ordnance Test Station

China Lake, Callformia

Attn: Code 753 (Library Div.)

Officer-in-Charge, Pasadena Annex

Naval Ordnance Teat Station

3203 E. Foothill Blvd.

Pasaitena 8, California

Attn: Codes P804 (Guid. \& Control Div.) P807 (Appl. Mech. Br.) P8074 (Hydro. Br.)

Comanding offlcer

U.S. Naval Underwater Ordnance Station liemport, Rhode Island

Commanding officer and Director

U.S. Naval Engineering Experiment station Annapolis, Maryland

Superintendent

U.S. Navel Eostgraduate School

Nonterey, California

Attn: Librartan

Superintendent

U.S. LTaval Acaderny

Annapel1s, Marylana

Attn: Ifbrarian

Commanding offlcer and Director

U.S. Navy Electronies Laboratory

San Diego 52, Califormla

Attn: Mr. C. M1ler

Commanding Officer and Director

U.S. Navy Underrater Sound Laboratory

Fort Trumbull, liew London,

Connecticut

Attn: 'r. IJ.A. Von binkle

Alr Force Offlce of Scientific Research Mecharics Division

Washington 25, D.C.

Conmiender

Alr Force Cernbridge Research Center

230 Albany Street

Cambridge 39, liassachusetts

Attn: Geophysical Research Library

Commanding officer

offlce of Ordnance Research

Box G1, Duke Station

Durhas, North Carolina

Director of Research

National Advisory Committee for

Aerorautica

1512 E Street, N.W.

Washington 25, D.C.

\section{Director}

Laugley Aeronautical Laboratory

National Advisory Committee for

Aeronautics

Langley fleld, Virglnia

Director

Amea Aeronautical Laborator

Vational Advisory Comittee for

Aeronautics

Hoffett Field, California
Director

Lew1s Flight Propulsion Laboratory

lational Adrisory Conmittee for

Aeronautics

cleveland, 11, ohto

National Bureau of Standard

Jaslungton 25, D.C.

Attn: Fluid Pechantes Section Dr. G.B. Schubauer Dr. P.S. Klebanoff Aerodynamics Section Dr. C.H. Tchen

Armed Services Technicai Information Agency Document Service Center Arlington tall Station Arlington 12, Vircinla

Office of Technical Services Department of Cormerce Washington 25, D.C.

Institute of lathematical sciences Ilew York Uni versity 25 Waverly Place

New York 3, New York

Attn: Frof. R. Courant Dr. F. Kraichnar

Prof. W.J. Pierson, Jr.

Oceanography Department

Wev York Uni versity

University Heights

New York 53, Hew Yor:

The John Hopkins University

Baltimore 18, liaryland

Attn: Mech. Pngre. Dept.

Prof. S. Corsin

Prof. 0.1\%. Phillips

Aero. Dept.

Prof. F.H. Clauser

Prof. L.S.G. Kovasznay

C1vil Engrg. Dept.

Prof. R.R. Lons

Institute for Fluid Dynamies and

Apolied lathenatics

Uni versity of l'aryland

College Park, laryland

Attn: Prof. J.ll. Burgers Frof. F.R. Jame

Division of Applied Mathenatics

Brom University

Providence 12, Rhode Island

Attn: Prof. 1. Holt

Dr. R. E. Ifeyer Dr. W.II. Reid

Harvand University

Cambridge 38 , llassacnusetts

Attn: Engrg. and Appl. Phys. Div. Frof. H. is. Amons

Math. Dept.

Prof. G. Blrkhoff

Prof. S. Goldstein

Massachusetts Inst1 tute of Technology

Cambridire 39, "iessachusetts

Attn: Math. Dept.

Prof. C.C. Iin

Hytro. Lab.

Prof. A.T. Ippen

Prof. J.W. Daily 
University of luchigan

Ann, Arbor, $111 \mathrm{chl}$ gar

Attn: Aero. Pingrg. Dept.

Prof. A.ll. Kuethe

Prof. M.S. Uberol

Prof. W.1. W11marth

Appl. Hech. Inept. Prof. C.S. I1h

C1 vil Bugrg. Dept. Prof. V.I. st reeter

Hisv. Mrch. Dept. Prot. R. B. Couch

Dr. J. C. Johnson

Englaeerine Research Institute

Untverstty of "'ichigan.

Ann Arbor, IIf chigan

Iorg Institute of firitraulc Research

State University of Iow

Iowa C1ty, Iowa

Attr: Prof. H. Rouse

Prof. L. Landineber

Callfomla Institute of Technology

Pasadena 4 , Calfornia

Attn: Prof. C.B. :illlkan

rof. T.Y. Th

Frof. H.W. Lfemant

Prof. D.S. Coles

Hydro. Lab.

Unversity of Callfornda

Department of Englneeriag

Berleley 4, California

Attn: Prof. II.A. Hnstein

Prof. L. Grossman

Prof. J.V. Wehausen

Director

Experitiental Torlne Tant:

Stevers Institute of Technology

711 Hudison St reet

Hoboken, New Jersey

Director

Mden Fyclraulic Laboratory

Worcester Polytechnic Inst tute

Worcester, Massachusetts

Director

Ordnaice Research Laboratory

penngylvania State Uriversity

Univers1ty Park, Pennsylvani

Dr. E. Sharark

Ordnance Research Laboratory

Pennsylvania State University

University Park, Pennsylvaria

Director

St. Anthony Falls Iyrlraulic Laboratory

Uni versity of itinnesote

Huneapolis 14, linnesota

Drector

Scripps Inst1tution of

oceanography

University of Califomis

La Jolla, Celiformis
Director

Soodo llole Ocarnogrephic

Inst 1 tute

Hoods Hole, Hassachusetts

Drector

Applied Hysics Leboratory

The John lopkins university

Bú2l Georela Avenue

silver Spring, liaryland

Dr. Th. won farman

1051 S. Harengo Street

Fasmlena, Califomia

Irof. W. R. Seers, H rector

Craduate School of Aeronaut1cal

Enclueering

Comell Un1 versity

Ithaca, ley Yor's

Dr. J. \%. Rohertson

Lepartment of Theoretical and Apriled

lechanles

Colle

Uhiversity of Illinols

Urbana, Illinols

Irof". :. "l. Innte, Hend

Aeronautical Eirglneering Iepartment

Catholic University of America

Wasungton, D.C.

Prof. A. Ferri, Head

Aeronautical Englneering and Appliad

Ifochuiles Department

Dolytechnic Institute of Brooklyn

99 Livingston street

Brooklym 1, llew York

Profs. U.D. Hayes and L.I. Crocco

Dopartmont of Aoronautical Forineering

Princeton University

Princeton, lew Jerscy

Prof. G. Ruerti

Departnent of lechanlcal Engineerting

Case Inst1tute of Technolocy

cleveland, onlo

Dr. F. R. "loore

Comell Aeronautical Laboratory

Correll Research Foundation

Bax 235

Turfalo 21, Ilew York

irof. S. Chandraselthar

Yerlies Observetor

Wliliams B.y, Wisconsin

Iror. 11. iac

liathematics Departmeat

Cornell University

Ithaca, New Yort

Dr. J. Ienufer

Iet Provulsion Laboratory

Cilfornta Institute of Technolory

Fasadena, Californta
Trof. S. Fiskituz!

Iepertment of :'echarical Enclreering

Syracuse und versity

yracuse, Hew York

Dr. S. Klne

Lepartmert of "schen1cal id glneerine

Stanfor Mn1vercity

Starford, Call fornis

Inst1tuta of Aerophysics

University of Toronto

Toronto 5, Cararia

Frof. J. H. Jackson

Engineering Experiment Station

Geor-1a Institute of Technologr

Atlasta, Georcto

trof. T. R. G. Ecliert

Departinent of ifchentcal Trefneertiz

Univergity of : innesota

ifrneapolis 14, Mnresota

Dr. I. R. Var Drlest

I'issile Development Division

North American Aviation, Inc.

Downey, Callfornts

[r. I. Dyer

Bolt, 3e ranek and Netman, Inc.

50 l'oulton st reet

Cambrider, "iassachusetts

Colorado State und versity

Fort Collins

Colorado

Atta: Prof. J.E. Cerprak

Lept. of Civil Engineering

Hydronautics, Inc.

200 Monroe St.

Lockville, Ma.

Attn: Mr. Phll11p Eisenberg $\checkmark$. Barshall p Tulin

Uni versity of Cincinnati

Cincinnat 1 31, cto

Attn: Library, Lept. of Prosics

Western Electro-Acoustic Lab., Inc 11789 San Vicente BIvd.

Los Angeles 49 , California 
This book may be kept

FOURTEEN DAYS

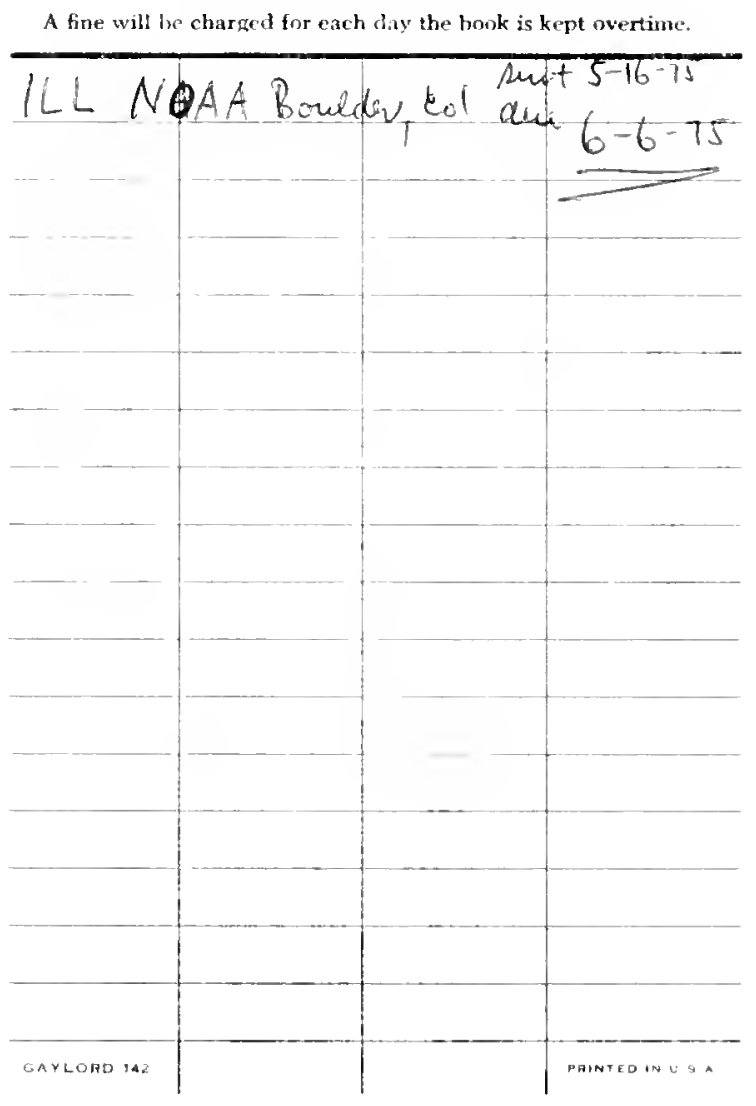




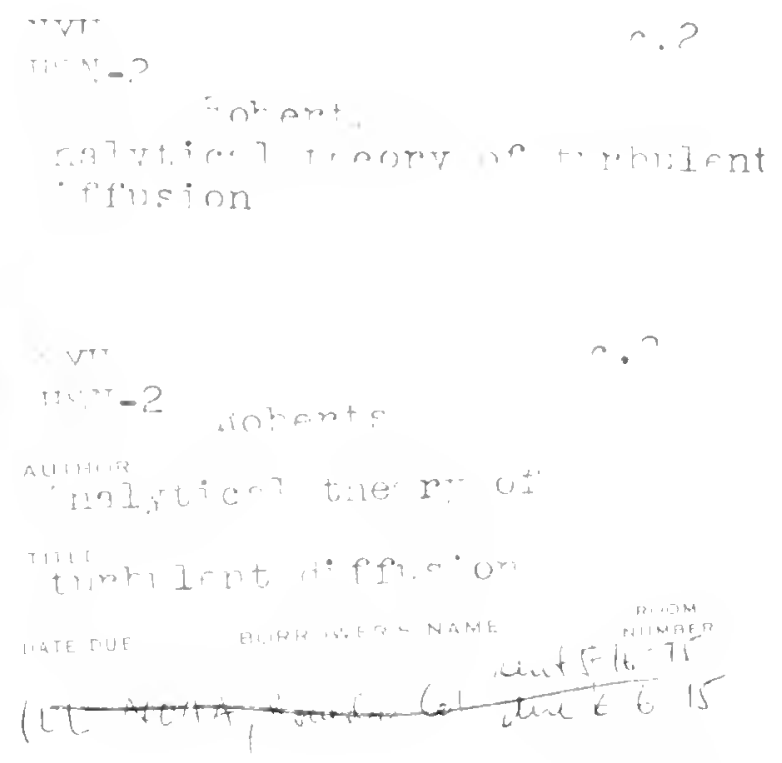

N. Y. U. Institute of

\section{Mathematical Sciences}

25 Waverly Place

New York 3, N. Y. 
